\title{
Investigation of the presence of charge order in magnetite by measurement of the spin wave spectrum
}

\author{
R. J. McQueeney, ${ }^{1, *}$ M. Yethiraj, ${ }^{2}$ W. Montfrooij, ${ }^{3}$ J. S. Gardner, ${ }^{4,5}$ P. Metcalf, ${ }^{6}$ and J. M. Honig ${ }^{6}$ \\ ${ }^{1}$ Department of Physics and Astronomy and Ames Laboratory, Iowa State University, Ames, Iowa 50011, USA \\ ${ }^{2}$ Center for Neutron Scattering, Oak Ridge National Laboratory, Oak Ridge, Tennessee 37831, USA \\ ${ }^{3}$ Department of Physics and Missouri Research Reactor, University of Missouri, Columbia, Missouri 65211, USA \\ ${ }^{4}$ Physics Department, Brookhaven National Laboratory, Upton, New York 11973, USA \\ ${ }^{5}$ NIST Center for Neutron Research, National Institutes of Standards and Technology, Gaithersburg, Maryland 20899, USA \\ ${ }^{6}$ Department of Chemistry, Purdue University, West Lafayette, Indiana 47907, USA
}

(Received 8 February 2006; published 8 May 2006)

\begin{abstract}
Inelastic neutron scattering results on magnetite $\left(\mathrm{Fe}_{3} \mathrm{O}_{4}\right)$ show a large splitting in the acoustic spin wave branch, producing a $7 \mathrm{meV}$ gap midway to the Brillouin zone boundary at $\mathbf{q}=(0,0,1 / 2)$ and $\hbar \omega=43 \mathrm{meV}$. The splitting occurs below the Verwey transition temperature, where a metal-insulator transition occurs simultaneously with a structural transformation, supposedly caused by the charge ordering on the iron sublattice. The wavevector $(0,0,1 / 2)$ corresponds to the superlattice peak in the low symmetry structure. The dependence of the magnetic superexchange on changes in the crystal structure and ionic configurations that occur below the Verwey transition affect the spin wave dispersion. To better understand the origin of the observed splitting, several Heisenberg models intended to reproduce the pair-wise variation of the magnetic superexchange arising from both small crystalline distortions and charge ordering were studied. None of the models studied predicts the observed splitting, whose origin may arise from charge-density wave formation or magnetoelastic coupling.

DOI: $10.1103 /$ PhysRevB.73.174409

PACS number(s): 75.30.Ds, 75.30.Et, 71.30. + h, 78.70.Nx
\end{abstract}

\section{INTRODUCTION}

Magnetite $\left(\mathrm{Fe}_{3} \mathrm{O}_{4}\right)$ was the first magnetic material to ever be discovered and utilized. It still has widespread usage in modern society as it is a rather strong permanent ferrimagnet $\left(T_{c}=858 \mathrm{~K}\right)$. While its magnetic properties are well known, magnetite surprisingly remains one of the more controversial examples of a correlated electron system. In 1939, Verwey discovered that magnetite undergoes a metal-to-insulator transition, resulting in a decrease of the conductivity by two orders of magnitude below the Verwey temperature, $T_{V}=123 \mathrm{~K} .{ }^{1}$ Verwey originally postulated that the hopping of extra electrons residing on the spinel B-site iron sublattice is responsible for the metallic conductivity. In the insulating phase, these extra electrons freeze out in an ordered pattern due to their mutual Coulombic repulsions. ${ }^{2}$ This charge ordering transition, called a Verwey transition, is one of the earliest instances of invoking many-body effects to explain a solid-state phase transition. In the intervening years, Verwey's hypothesis has survived in some shape or form. Neutron diffraction eventually demonstrated that the symmetry lowering predicted by Verwey's original charge ordering model cannot be entirely correct. ${ }^{3}$ Several other charge ordering schemes have been proposed that are consistent with the low-temperature monoclinic crystal symmetry. ${ }^{4,5}$ Due to the complexity of the low-temperature structure (224 atoms/unit cell) and twinning-related multiple monoclinic domains in the low-temperature phase, conclusive evidence for the existence of charge ordering is a difficult claim. So difficult, in fact, that new evidence is being put forth that raises serious doubts that magnetite is charge ordered at all at low temperatures, ${ }^{6}$ opening a new dialogue about this venerable system. ${ }^{7,8}$
One property that should be very sensitive to the presence of charge ordering is the spin wave spectrum. At room temperature, the spin wave dispersion is consistent with a simple nearest-neighbor Heisenberg Hamiltonian. The magnetic superexchange interactions between iron pairs is mediated by oxygen anions and have been determined by inelastic neutron scattering. ${ }^{9,10}$ If charge ordering is present below $T_{V}$, the superexchange values will be modified in a periodic way due to the charge (valence) ordering. This modification will appear as a splitting of the spin wave spectrum at wavevectors corresponding to the charge ordering wavevector.

In this paper, we report inelastic neutron scattering results that clearly show a large $(7 \mathrm{meV})$ splitting in the acoustic spin wave branch below $T_{V}$. The splitting occurs midway to the Brillouin zone boundary at $\mathbf{q}=(0,0,1 / 2)$ and $\hbar \omega=43 \mathrm{meV}$. The wavevector $(0,0,1 / 2)$ corresponds to the cell doubling supposedly caused by charge ordering. To better understand the origin of the splitting, we have constructed a series of Heisenberg models intended to reproduce the pairwise variation of the magnetic superexchange arising from both small crystalline distortions and charge ordering. We find that none of the models studied predicts the observed splitting. A preliminary report of the results of these measurements has been published elsewhere. ${ }^{11}$

\section{EXPERIMENT}

\section{A. Samples}

Above $T_{V}$, magnetite adopts a cubic inverse spinel crystal structure with $\mathrm{Fd} \overline{3} \mathrm{~m}$ space group and a lattice constant of $a_{c}=8.394 \AA$. The primitive rhombohedral unit cell consists of six iron atoms and eight oxygen atoms whose positions 
TABLE I. Atomic positions in magnetite at room temperature. The space group is $\mathrm{Fd} \overline{3} \mathrm{~m}$ (No. 227) using the second origin choice $(\overline{4} 3 \mathrm{~m})$. The cubic lattice parameter is $a_{c}=8.394 \AA$.

\begin{tabular}{ccccc}
\hline \hline Atom & Site & $d_{x}$ & $d_{y}$ & $d_{z}$ \\
\hline A & $8(\mathrm{a}), \overline{4} 3 \mathrm{~m}$ & 0 & 0 & 0 \\
B & $16(\mathrm{~d}), \overline{3} \mathrm{~m}$ & $5 / 8$ & $5 / 8$ & $5 / 8$ \\
$\mathrm{O}$ & $32(\mathrm{e}), 3 \mathrm{~m}$ & 0.379 & 0.379 & 0.379 \\
\hline \hline
\end{tabular}

are given in Table I. There are two different symmetry iron sites, labeled A and B. The two A sites reside in tetrahedrally coordinated oxygen interstices, the four B sites have octahedral coordination. From valence counting, the A site is $3^{+}$ ( $3 d^{5}$ electron configuration), and the $\mathrm{B}$ site has a fractional average valence of $2.5^{+}$. The magnetic structure is that of a collinear ferrimagnet with $\mathrm{A}$ and $\mathrm{B}$ moments aligned antiparallel.

Below $123 \mathrm{~K}$, magnetite undergoes a first-order metalinsulator transition. The transition lowers the crystallographic symmetry from cubic to the monoclinic Cc space group. The monoclinic structure consists of slight distortions from a superstructure of the cubic cell of dimensions $\sqrt{2} a_{c}$ $\times \sqrt{2} a_{c} \times 2 a_{c}\left(a_{m}=11.868 \AA, b_{m}=11.851 \AA, c_{m}=16.752 \AA\right.$, and $\left.\beta=90.2^{\circ}\right)$. There are 32 formula units in the Cc cell and a table of the atomic positions can be found in Ref. 4. Ignoring the small monoclinic distortion, the low-temperature structure can also be described in a slightly less complicated orthorhombic structure in the Pmca space group. This is related to a cubic supercell of size $a_{c} / \sqrt{2} \times a_{c} / \sqrt{2} \times 2 a_{c}$. There are eight formula units in the Pmca cell and the atomic positions are listed in Ref. 12. There are no reports to indicate that the magnetic moments are non-collinear below $T_{V}$. In our discussion of the low-temperature phases, we will refer to either the $\mathrm{Cc}$ or Pmca cell as the need warrants. As the atomic distortions in the Verwey structure are very small, throughout the paper we will only refer to crystal directions in terms of the $(h, k, l)$ indices of the original cubic cell, to avoid confusion.

We used various single-crystal samples weighing from $5-10 \mathrm{~g}$ each that were prepared from powdered material by use of a radio-frequency induction melting technique. ${ }^{13}$ This method permits melting of $\mathrm{Fe}_{3} \mathrm{O}_{4}$ in a crucible lined with a solid of the same composition, so as to minimize accidental contamination of the melt. After slow cooling, the boule was fractured and single crystals were extracted. These crystals were then reannealed in specialized equipment ${ }^{14}$ under appropriate $\mathrm{CO}_{2} / \mathrm{CO}$ atmospheres, so as to achieve the desired oxygen/iron ratio. The Verwey transition temperature for these samples is $\sim 123 \mathrm{~K}$ with a narrow transition width; a good indication that the samples are homogeneous, with few metallic impurities, and nearly ideal oxygen stoichiometry. ${ }^{15}$ In addition, ideal oxygen stoichiometry is indicated by the observation of a $0.3^{\circ}$ splitting due to monoclinic domains. ${ }^{15}$ Crystal mosaics were $\sim 0.1^{\circ}$ as obtained from neutron diffraction rocking curves.

\section{B. Triple-axis measurements}

We performed triple-axis neutron scattering measurements of the magnetic excitations using several different instruments under a variety of experimental configurations. Triple-axis measurements were made on the HB-3 and HB-1 spectrometers at the High Flux Isotope Reactor at Oak Ridge National Laboratory and the C-5 spectrometer at the National Research Universal (NRU) reactor at Chalk River Laboratories. Detailed experimental configurations are shown in Table II and will be referred to in the text by the configuration label. Measurements were made in a horizontal magnetic field as well as a zero-applied magnetic field. The horizontal field is applied along the cubic [001] direction. The applied magnetic field served two purposes: (1) A single magnetic domain sample can be created in a modest magnetic field of $H<1 \mathrm{~T}$, and (2) in the low-temperature phase, field cooling with $H \sim 1 \mathrm{~T}$ will cause the cell doubling direction (the $c$ axis of the monoclinic structure) to orient along the applied field direction. ${ }^{3}$ Without an applied field, the three equivalent cubic axes become the $c$ axis of the monoclinic phase, giving three domains. In addition, there are two monoclinic domains for each cubic domain. However, the two domains are only separated by $0.3^{\circ}$ and are treated as a single domain for the inelastic scattering measurements.

\section{Observation of the gap}

Above the Verwey transition, the spin wave dispersion can be well understood as a simple Heisenberg ferrimagnet. The dispersion consists of six branches; one acoustic and five

TABLE II. Instruments and configurations used for measurements. The C-5 instrument is located at NRU at Chalk River Laboratories. HB1 and HB3 instruments are located at the High Flux Isotope Reactor at Oak Ridge National Laboratory. Each configuration has a fixed final energy. Collimations are reported as full width at half maximum in minutes of arc. Throughout the paper, configurations will be referred to by the configuration label in the first column.

\begin{tabular}{cccccc}
\hline \hline Label & Instrument & $\begin{array}{c}\text { Magnetic } \\
\text { field }\end{array}$ & $E_{f}(\mathrm{meV})$ & $\begin{array}{c}\text { Monochromator/ } \\
\text { analyzer }\end{array}$ & $\begin{array}{c}\text { Horizontal } \\
\text { collimation }\end{array}$ \\
\hline A & C-5 & Horizontal & 14.3 & $\mathrm{Be}(002) / \mathrm{PG}(002)$ & $36^{\prime}-30^{\prime}-48^{\prime}-120^{\prime}$, \\
Collimation
\end{tabular}




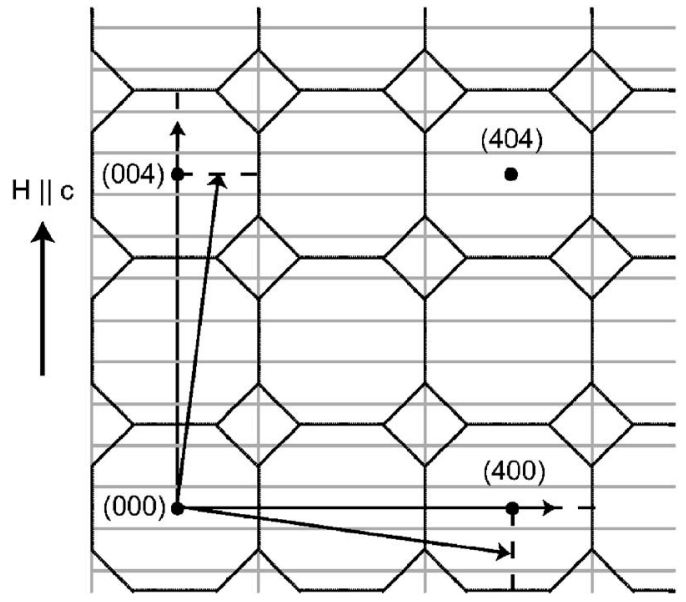

FIG. 1. Scattering plane in horizontal field configuration with a field of $1.1 \mathrm{~T}$ applied along the cubic [001] direction. Black (and gray) lines show the Brillouin zone boundaries of the cubic $\left(T>T_{V}\right)$ and orthorhombic $\left(T<T_{V}\right)$ lattices, respectively. Arrows indicate typical scattering vectors studied. The reduced wavevectors are equivalent in the cubic phase. In the orthorhombic phase, the cell doubling axis lies along the field and $[100] /[001]$ directions are no longer equivalent.

optical. Brockhouse originally measured the acoustic branch and one optical branch, ${ }^{9}$ and these dispersion data were fit to a Heisenberg model with nearest-neighbor superexchange parameters $J_{A B}=-4.8 \mathrm{meV}$ and $J_{B B}=0.48 \mathrm{meV} .{ }^{10}$ We have performed measurements of the acoustic dispersion along the [001] direction which are consistent with Brockhouse's original work above $T_{V}$. This will be discussed in more detail below. At temperatures below the Verwey transition, we observe clear evidence of the splitting of the acoustic branch at $\mathbf{q}=(0,0,1 / 2)$ (in units of $2 \pi / a_{c}$ referencing the cubic cell). We define the reduced wavevector $\mathbf{q}$ as that part of the momentum transfer (scattering) vector $\hbar \mathbf{Q}$ to lie within the first Brillouin zone such that $\mathbf{Q}=\boldsymbol{\tau}+\mathbf{q}$, where $\boldsymbol{\tau}$ is a reciprocal lattice vector of the cubic structure.

The clearest indication of the nature of the gap is seen in a scattering configuration with a horizontal magnetic field of 1.1 Tesla applied along the cubic [001] direction. Measurements were made on the C-5 spectrometer at Chalk River Laboratories in configuration A of Table II. Figure 1 shows the orientation of the magnetic field in the $[h 0 l]$ scattering plane, typical measured momentum transfer vectors, and the Brillouin zone boundaries of both the cubic $\left(T>T_{V}\right)$ and orthorhombic $\left(T<T_{V}\right)$ crystal structures. Figure 2 shows constant-Q energy scans with momentum transfers $\mathbf{Q}=(0,0,4.5),(4.5,0,0),(4,0,1 / 2)$, and $(-1 / 2,0,4)$ each corresponding to cubically equivalent $(0,0,1 / 2)$-type reduced wavevectors with an acoustic spin wave energy of $\hbar \omega=43 \mathrm{meV}$. In the orthorhombic phase, the $\mathbf{q}=(0,0,1 / 2)$ and $(1 / 2,0,0)$ reduced wavevectors are inequivalent, as is clear from Fig. 1, with $(0,0,1 / 2)$ being a Brillouin zone center of the orthorhombic structure. The acoustic spin wave excitation is clearly split below $T_{V}$ into two distinct modes at 39 and $46 \mathrm{meV}$ for $\mathbf{q}=(0,0,1 / 2)$ [Figs. 2(a) and 2(b)]. Brockhouse and co-authors ${ }^{15}$ had previously studied the dis-

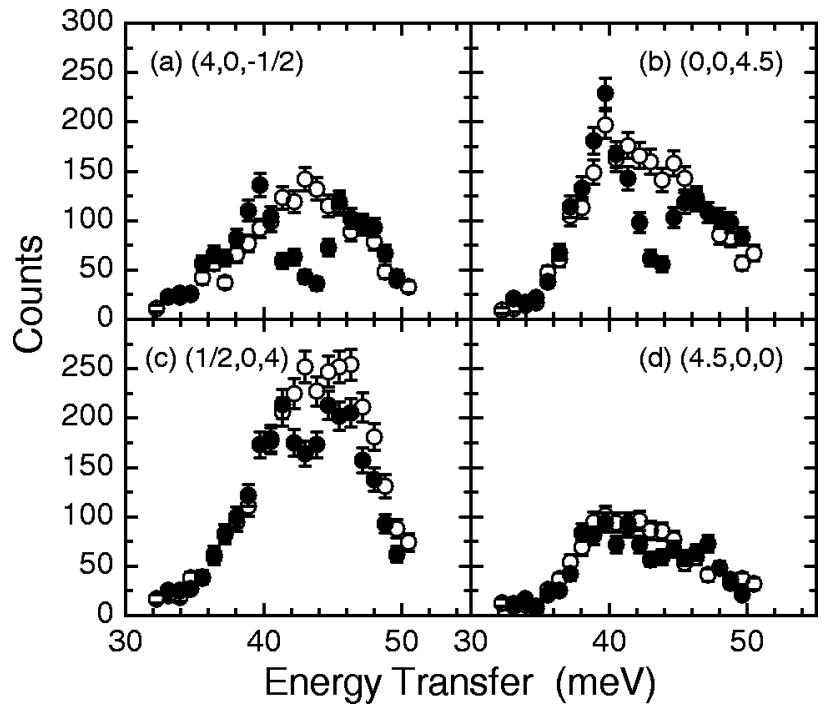

FIG. 2. Observed scattering from the acoustic spin wave at four cubically equivalent $(0,0,1 / 2)$-type reduced wavevectors (a) $(4,0$, $-1 / 2)$, (b) $(0,0,4.5),(\mathrm{c})(1 / 2,0,4)$, and (d) $(4.5,0,0)$ at $T=130 \mathrm{~K}$ (cubic, empty circles) and $T=115 \mathrm{~K}$ (monoclinic, filled circles). Measurements were made in configuration $\mathrm{A}$ in a horizontal magnetic field along the cubic [001] direction. The $(0,0,1 / 2)$ and $(1 / 2,0,0)$ reduced wavevectors are inequivalent in the Verwey phase. The spin wave excitation is split below $T_{V}$ at the $(0,0,1 / 2)$ position $[(a)$ and (b)], but only weakly split along $(1 / 2,0,0)$ $[(\mathrm{c})$ and $(\mathrm{d})]$ demonstrating that splitting occurs primarily along the cell doubling direction. Spin wave intensity is approximately twice as large for wavevectors along [001] [(b) and (c)] than along [100] $[(\mathrm{a})$ and (d)] due to the neutron spin cross section.

persion below $T_{V}$, but reported no substantive change compared to the room-temperature dispersion. ${ }^{15,16}$ We assume that this negative result was due to the use of natural crystals where impurities can have deleterious effects on the Verwey transition. ${ }^{7}$ Using high-quality synthetic single crystals, we observe a rather large splitting of the acoustic branch below $T_{V}$. Weak evidence for splitting can also be seen for $\mathbf{q}=(1 / 2,0,0)$ [Figs. $2(\mathrm{c})$ and $2(\mathrm{~d})]$. The main splitting therefore occurs only when $\mathbf{q}$ is along the cell doubling direction of the orthorhombic structure. The small residual splitting remaining at the $(1 / 2,0,0)$ wavevectors may be due to the incomplete removal of domains causing a minority of cell doubling domains to occur along the [100] direction. It is also clear that the two excitation peaks below $T_{V}$ appear narrower than the single peak above $T_{V}$. We will address the intrinsic widths versus resolution widths for these peaks in Sec. III.

Figure 2 also shows that the spin wave intensity is approximately twice as large for $\mathbf{Q}$ along [001] [Figs. 2(b) and 2(c)] than along [100] [Figs. 2(a) and 2(d)]. This is due to the vector nature of the neutron spin scattering cross section. The scattered intensity for a ferrimagnet aligned in a magnetic field along the $z$ direction is

$$
S(\mathbf{Q}, \omega) \propto\left(1+\frac{Q_{z}^{2}}{|\mathbf{Q}|^{2}}\right) .
$$

Measurements with the scattering vector lying along the field direction will have twice the signal of a scattering vector 


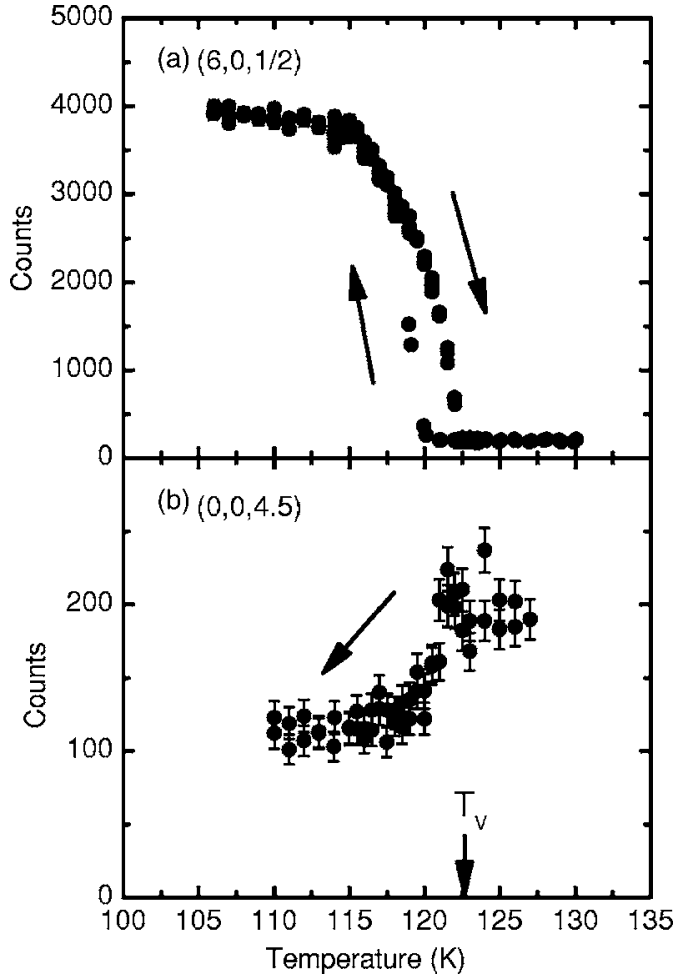

FIG. 3. Temperature dependence of (a) the $(6,0,1 / 2)$ superlattice peak, and (b) the acoustic spin wave intensity at $\mathbf{Q}=(0,0,4.5)$ and $\hbar \omega=43 \mathrm{meV}$ measured in configuration $\mathrm{B}$. The appearance of the superlattice peak below $T_{V}$ occurs simultaneously with the splitting of the spin wave branch. Slanted arrows indicate cooling/warming.

perpendicular to the field, as is clear from Fig. 2.

The spin wave splitting occurs only below $T_{V}$. Figure 3(b) shows the temperature dependence of the intensity in the gap at $\mathbf{q}=(0,0,1 / 2)$ and $\hbar \omega=43 \mathrm{meV}$. This measurement was performed using configuration $\mathrm{B}$ without a horizontal magnetic field. Below $T_{V}$, the sample contains all three orthorhombic $c$-axis domains; hence, the ratio of intensities measured at the gap energy above and below $T_{V}$ is not as large as Fig. 2(a). The gap begins to form below the Verwey temperature, and can be compared to the order parameter of the $(6,0,1 / 2)$ superlattice peak in Fig. 3(a).

\section{Extent of the gap in q space}

Figure 4 displays constant-Q energy scans along the [001] direction as measured in configuration C. Figure 4(a) shows a set of scans above the Verwey transition at room temperature, and Fig. 4(b) shows scans along the same direction at $T=115 \mathrm{~K}$. As configuration $\mathrm{C}$ has no applied magnetic field, the resulting spin waves observed along [001] at $T=115 \mathrm{~K}$ are actually averaged over all three orthorhombic principal directions. Similar to Fig. 3(b), the gap is still observed despite the domain averaging. In each figure, the peaks of the spin wave excitations are marked with a gray circle. The spin wave gap is indicated by the red slanted line and appears to have some $\mathbf{q}$ dependence. The dotted line indicates the gap energy of $43 \mathrm{meV}$ at $(0,0,4.5)$. Phonon excitations were also observed and are indicated by the dashed lines. Precise fits to

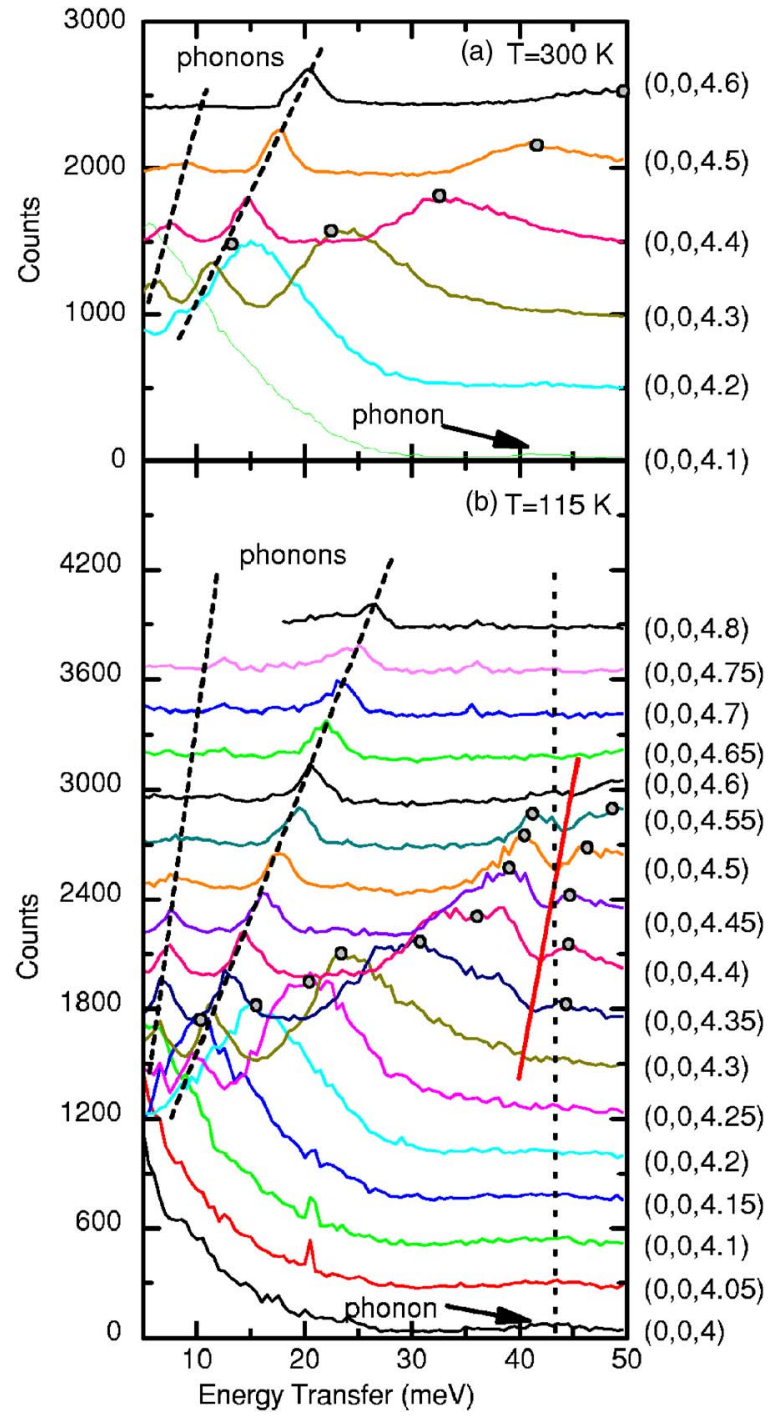

FIG. 4. (Color online) Constant-Q energy cuts made for several Q-vectors along the [001] direction at (a) $300 \mathrm{~K}$ and (b) $115 \mathrm{~K}$, in configuration $\mathrm{C}$. This instrument configuration has no applied magnetic field, thus the spin waves excitations below $T_{V}$ are averaged over all three orthorhombic directions. The gap is still clear despite this and is indicated by the slanted red line in (b) and the dotted line marks the gap energy at $(0,0,4.5)$ and $T=115 \mathrm{~K}$. In each figure, gray circles mark the spin wave peaks. The dashed lines and arrow indicate phonon excitations.

the low-temperature dispersion will be discussed in Sec. III, as the resolution effects are important in the interpretation of the data.

The HB1 and HB3 spectrometers were used to study the extent of the splitting in other directions in reciprocal space. Using the $\mathrm{B}$ and $\mathrm{D}$ configurations, a series of constant energy scans were measured on the $[h, 0,4+l]$ plane above and below the Verwey transition. Mesh scans were performed at $\hbar \omega=39 \mathrm{meV}, 43 \mathrm{meV}$, and $46 \mathrm{meV}$, the energy positions of the lower peak, the gap, and the upper peak of the split mode at $(0,0,1 / 2)$. Figure 5 shows false color contour plot of the scattered intensity and Fig. 6 shows line plots of the constant energy cuts along the $(h, 0,4.5)$ direction. Above the transi- 


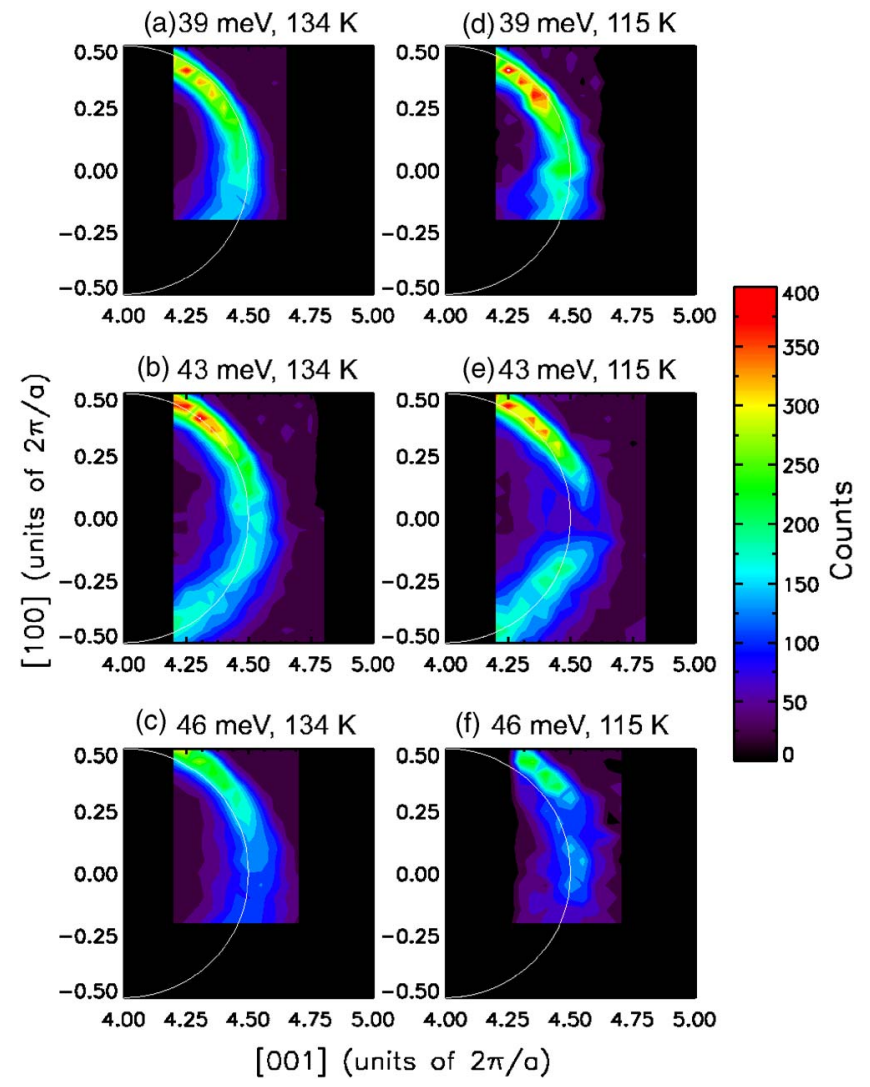

FIG. 5. (Color online) Constant energy contour plots of the scattering in the [h01] plane near the (004) Brillouin zone above $T_{V}$ [ $T=134 \mathrm{~K}$, left panels, (a), (b), and (c) $]$ and below $T_{V}[T=115 \mathrm{~K}$, right panels (d), (e), and (f)]. Mesh scans performed at constant energies of $39 \mathrm{meV}$ [top row, (a) and (d)], $43 \mathrm{meV}$ [middle row, (b) and (e)], and $46 \mathrm{meV}$ [bottom row, (c) and (f)] were used to construct the contour plots. These energies correspond to the lower peak, gap, and upper peak of the low-temperature split spin wave excitation at $(0,0,1 / 2)$. Measurements were performed in the D configuration with the HB1 spectrometer in zero-applied field.

tion, the contour plots demonstrate that the spin wave dispersion is isotropic and forms a circular ring of intensity around the (004) Brillouin zone center. In the Verwey phase, the gap at $43 \mathrm{meV}$ is clearly seen and extends $+/-0.1-0.2$ reciprocal lattice units (rlu) in the transverse direction along [100]. At $39 \mathrm{meV}$, it does not appear that the spin waves are affected to any great extent. At $46 \mathrm{meV}$, a suppression of the intensity is observed near the $(0.25,0,4.5)$ position at low temperatures, as shown in Figs. 5 and 6. This feature is not another gap, but is rather the original gap at $(0,0,4.5)$ picked up by the experimental resolution of the instrument. We will delay further discussion of important resolution effects until the next section, where we consider the effect of experimental resolution on the interpretation of the gap structure.

\section{DATA ANALYSIS AND MODELING}

Before making a detailed analyses of the dispersion data, we introduce model calculations for the spin wave scattering cross section for magnetite in the cubic spinel phase. This

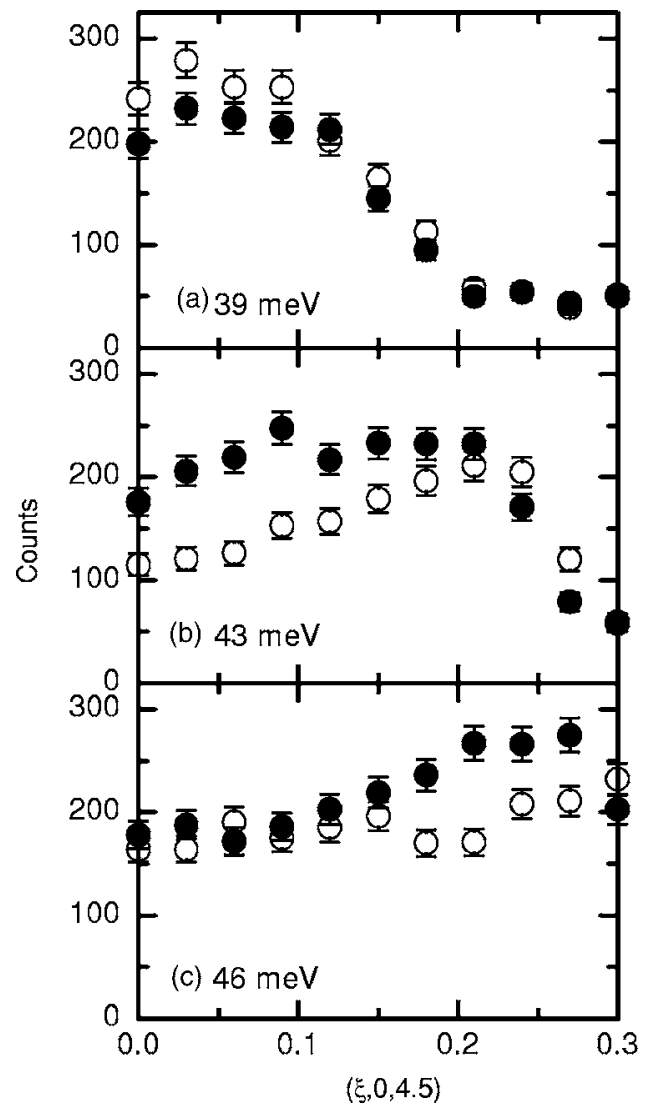

FIG. 6. Constant energy cuts along the $(h, 0,4.5)$ direction above $T_{V}(T=150 \mathrm{~K}$, filled circles $)$ and below $T_{V}(T=110 \mathrm{~K}$, empty circles) at (a) $\hbar \omega=39 \mathrm{meV}$, (b) $\hbar \omega=43 \mathrm{meV}$, and (c) $\hbar \omega=46 \mathrm{meV}$. Measurements were performed with the B configuration on the HB3 spectrometer in zero-applied field.

model can be used to perform resolution convolutions of the spin wave cross section for comparison to the data. Once the machinery is established, its use can be extended to the more complicated low symmetry phase occurring below $T_{V}$. We start with a discussion of the local ionic states and build up to the collective spin excitations.

\section{A. Magnetic states and excitation spectrum of magnetite}

Following primarily the work of Buyers et al., ${ }^{17}$ the general Hamiltonian for the lowest-energy magnetic states of the iron atoms in magnetite can be written as

$$
H=H_{\text {Hund }}+H_{\mathrm{CF}}+H_{\mathrm{SO}}+H_{\mathrm{ex}},
$$

where $H_{\text {Hund }}$ describes the intra-atomic electronic correlations, $H_{\mathrm{CF}}$ is the crystalline electric field, and $H_{\mathrm{SO}}$ is the spin-orbit coupling in the Fe $3 d$ orbitals. $H_{\mathrm{ex}}$ is the exchange interaction between atoms. The Hamiltonian can be further categorized as containing two terms $H=H^{(1)}+H^{(2)} . H^{(1)}$ is a single-ion term:

$$
H^{(1)}=H_{\mathrm{Hund}}+H_{\mathrm{CF}}+H_{\mathrm{SO}}+H_{\mathrm{mf}},
$$

where $H_{\mathrm{mf}}$ describes the molecular field arising from ferrimagnetic long-range order. The residual term containing 
exchange interactions between the ions, $H^{(2)}$, will describe the spin waves in the ordered state

$$
H^{(2)}=H_{\mathrm{ex}}-H_{\mathrm{mf}} .
$$

\section{B. Single-ion term}

In magnetite, the strength of each term in $H^{(1)}$ has the following order: $H_{\mathrm{Hund}} \gg H_{\mathrm{CF}} \gg\left(H_{\mathrm{SO}}, H_{\mathrm{mf}}\right)$. In this weak-field limit, we consider only the ground-state term of the Hund multiplet, with higher terms being several $\mathrm{eV}$ above the Hund's rule ground state. The crystal field is the next strongest term in the Hamiltonian, and will split the ground-state Hund's rule term. The two remaining interactions are generally weaker than the crystal field and will determine the details of the ionic configuration.

In the high-temperature cubic inverse spinel structure of magnetite, there are two crystallographically distinct sites; The tetrahedral A site and the octahedral B site. The A sites contain only $\mathrm{Fe}^{3+}$ ions with a $3 d^{5}$ electronic configuration, giving a singlet ${ }^{6} S_{5 / 2}$ Hund's ground-state term. The point group symmetry of the A site is cubic $\left(T_{d}\right)$; however, this crystalline electric field cannot not split the orbital singlet ground state. The ground state also has no spin-orbit splitting, since the orbital moment is zero. Therefore, $\mathrm{Fe}^{3+}$ has a spin-only ground state $S=5 / 2$.

The $\mathrm{B}$ sites contain both $\mathrm{Fe}^{3+}$ and $\mathrm{Fe}^{2+}$ states. Similar to the A-site, the B-site $\mathrm{Fe}^{3+}$ Hund's rule singlet ground state is unsplit by crystal-field and spin-orbit interactions and has a $S=5 / 2$ ground state. On the other hand, the $\mathrm{Fe}^{2+}$ ion has a ${ }^{5} D_{4}$ ground state that will be split by the crystal field and spin-orbit interactions. The B site actually possesses a trigonal $\left(D_{3 d}\right)$ point group symmetry in the cubic $\mathrm{Fd} \overline{3} \mathrm{~m}$ space group $\left(H_{\mathrm{CF}}=H_{\text {cubic }}+H_{\text {trigonal }}\right)$. The trigonal component of this field $\left(H_{\text {trigonal }} \sim 150 \mathrm{meV}\right)$, which is due to neighbors beyond the first shell, is weak in comparison to the nearest-neighbor oxygen octahedral field $\left(H_{\text {cubic }} \sim 1.5 \mathrm{eV}, H_{\text {trigonal }} \ll H_{\text {cubic }}\right){ }^{18}$ The larger octahedral crystal field splits the ${ }^{5} D_{4}$ ground state into a triply degenerate ground state $\left({ }^{5} T_{2}\right)$ and an excited state doublet $\left({ }^{5} E\right)$, with the ${ }^{5} E$ state sufficiently high in energy that we may disregard it from now on. The weaker trigonal field will further split the cubic ${ }^{5} T_{2}$ ground state into a ground-state singlet $\left({ }^{5} A_{1}\right)$ and excited-state doublet $\left({ }^{5} E\right)$. For cubic symmetry, with the trigonal axis $\alpha \approx 60^{\circ}$, only minor mixing occurs between the two ${ }^{5} E$ states. ${ }^{19}$ Spin-orbit interactions are expected to be even smaller than the trigonal field $\left(E_{\mathrm{SO}} \sim 10 \mathrm{meV}\right),{ }^{18,20,21}$ splitting the ${ }^{5} E$ orbital doublet into five doubly degenerate $|l s\rangle$ states and leaving the ${ }^{5} A_{1}$ orbital singlet ground state unchanged.

Finally, we consider the molecular field acting on the both the $\mathrm{Fe}^{2+}$ and $\mathrm{Fe}^{3+}$ ions. At room temperature and below, we can assume that the magnetization is nearly saturated in the ferrimagnetic state with $T_{C}=858 \mathrm{~K}$. The molecular field at low temperatures can then be estimated from the Curie constants on each sublattice, the Curie temperature $\left(T_{C}\right)$, and the saturation magnetization $\left(M_{s}\right)$. The molecular field is

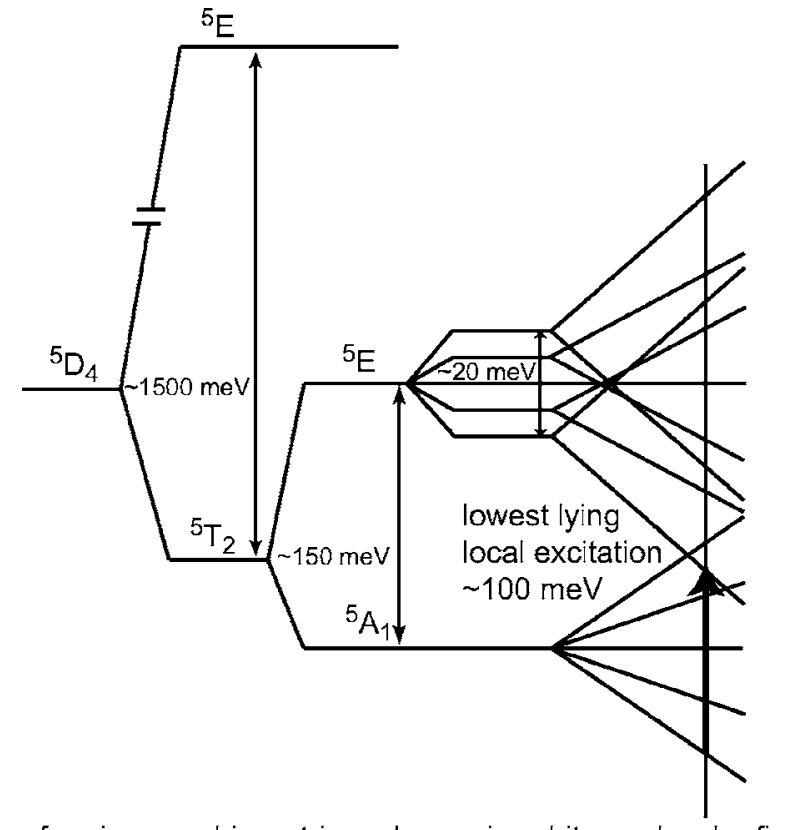

free ion cubic trigonal spin-orbit molecularfield

FIG. 7. Schematic drawing of the local electronic states for an $\mathrm{Fe}^{2+}$ ion on the $\mathrm{B}$ site in the cubic phase of magnetite. The free ion term is split by strong cubic crystal field and a relatively weaker trigonal field, resulting in an orbital singlet ground state. This orbital singlet is unsplit by weak spin-orbit interactions; however, the doublet excited state is split by this interaction. Subsequent symmetry lowering due to the Verwey transition cannot split the orbital singlet ground state. All states are subjected to Zeeman splitting by the molecular field. Given an approximate value of $350 \mathrm{~T}$, the Zeeman splittings can lead to low lying local excitations of $\sim 100 \mathrm{meV}$. The lowest dipole excitation of local character that is observable by neutron scattering is $\sim 200 \mathrm{meV}$. The local excitations are well above the energies considered here.

$$
B_{\mathrm{mf}}=\frac{T_{c}}{\sqrt{C_{A} C_{B}}} M_{s},
$$

where the Curie constants are

$$
C_{i}=\frac{N_{i} g_{i}^{2} J_{i}\left(J_{i}+1\right) \mu_{B}^{2}}{3 k_{B}} .
$$

For magnetite with $M_{s}=510 \mathrm{G}, T_{C}=858 \mathrm{~K}, C_{A}=0.1 \mathrm{~K}\left(J_{A}\right.$ $\left.=5 / 2, g_{A}=2\right)$, and $C_{B} \approx 0.16 \mathrm{~K}\left(J_{B} \approx 2.25, g_{B} \approx 2\right)$ gives a molecular field of $\sim 350 \mathrm{~T}$. For the $\mathrm{Fe}^{3+}\left(\mathrm{Fe}^{2+}\right)$ ions, the maximum Zeeman splitting in the molecular field is $g S \mu_{B} B_{\mathrm{mf}} \approx 100 \mathrm{meV}(80 \mathrm{meV})$. Figure 7 shows a schematic drawing of the spectrum of single-ion states in cubic magnetite.

The above treatment of the local electronic states is strictly for an insulator. We have ignored that the cubic phase is poorly metallic, with electronic bandwidths of order $1 \mathrm{eV} .{ }^{22,23}$ Therefore, the electronic hybridization will mix the trigonal crystal field ground states, thereby returning some orbital degeneracy to the B-site iron atoms.

Below $T_{V}$, the monoclinic distortion will lower the point group symmetry of each iron site to either $1, \overline{1}$, or $\mathrm{m}$, and 
open a gap in the electronic density of states. In the cubic spinel phase, the local ground-state electronic configurations for $\mathrm{Fe}^{2+}$ or $\mathrm{Fe}^{3+}$ ions are all orbital singlets. This symmetry lowering cannot split any of the ground-state ionic configurations, but it will split the ${ }^{5} E$ excited state by a small amount (of order $10 \mathrm{meV}$ ). Therefore, we do not expect the monoclinic distortion to produce any additional low-energy crystal field excitations ( $\leqslant 150 \mathrm{meV}$ ) on any of the iron sites.

Using these arguments, we can assume that there are no low-energy $(\$ 100 \mathrm{meV})$ crystalline electric field or spinorbit excitations either in the cubic or monoclinic phase. These local states have no orbital freedom and can be treated as spin variables in a Heisenberg treatment of the collective excitations.

\section{Collective spin wave excitations}

Starting from the local orbitally quenched ionic configurations, we can now study the low-energy collective spin wave excitations given by $H^{(2)}$. A general Heisenberg Hamiltonian is chosen to represent the interactions between the local moments. In the absence of an applied magnetic field, the Heisenberg Hamiltonian is given by

$$
H^{(2)}=-\sum_{\left\langle l i, l^{\prime} j\right\rangle} J\left(l i ; l^{\prime} j\right) \mathbf{S}_{l i} \cdot \mathbf{S}_{l^{\prime} j}
$$

where $J\left(l i ; l^{\prime} j\right)$ are the pair-wise exchange values between ground-state configurations of the $i$ th atom in the $l$ th unit cell and the $j$ th atom in the $l^{\prime}$ th unit cell. The sum is over distinct pairs only. Single-ion anisotropy terms are extremely small in magnetite (with anisotropy fields of $0.1-1 \mathrm{~T}$, depending on temperature) and are set to zero. ${ }^{10,24,25}$ For an arbitrary number of collinear spins in the unit cell, Saenz ${ }^{26}$ has developed a formalism to calculate the spin wave excitation energies, eigenvectors, and neutron scattering intensities. $\mathbf{S}_{l i}$ is the vector spin operator for the ground-state ion with spin magnitude $\sigma_{i} S_{i}$, where $S_{i}$ is positive and $\sigma_{i}= \pm 1$ with $+1(-1)$ parallel (antiparallel) to the $z$-quantization axis. For the ferrimagnetic magnetite structure, $\sigma_{\mathrm{A}}=-1$ and $\sigma_{\mathrm{B}}=+1$. After performing the Holstein-Primakoff transformation, the secular equation for the system is $(\underline{\mathbf{M}}-\gamma \underline{\mathbf{I}}) \mathbf{T}=0$ where

$$
M_{i j}(\mathbf{q})=\delta_{i j} \sum_{l k} J(0 i ; l k) \sigma_{k} S_{k}-\sigma_{j} \sqrt{S_{i} S_{j}} \sum_{l} J(0 i ; l j) \exp (i \mathbf{q} \cdot \mathbf{l}) .
$$

The eigenvalues, $\gamma_{n}(\mathbf{q})$, and eigenvectors, $T_{n}(\mathbf{q})$, are obtained by the diagonalization of the secular matrix $\underline{\mathbf{M}}(\mathbf{q})$ at wavevector $\mathbf{q}$, where $n$ labels the spin wave branch. Inspection of the matrix shows that it is not Hermitian, due to $\sigma_{j}$. However, the eigenvalues for this matrix are purely real and an entire branch must either be entirely positive or entirely negative (i.e., no branch ever crosses zero), with the number of negative branches equal to the number of antiparallel spin sites. Thus, the spin wave dispersion for branch $n$ is $\hbar \omega_{n}(\mathbf{q})=\left|\gamma_{n}(\mathbf{q})\right|$. The normalized eigenvector has components

$$
T_{n i}=\sqrt{S_{i}} \xi_{n i} / \sqrt{\sum_{i} S_{i} \xi_{n i}^{2}}
$$

where $\xi_{n i}{ }^{2}$ is the fraction of the $i$ th spin contained in the eigenvector and $\Sigma_{i} T_{n i}^{2}=1$ for each branch. This definition of the eigenvector is used to calculate the neutron cross section.

\section{Neutron scattering cross-section for spin waves}

The neutron cross section for unpolarized magnetic scattering is

$$
\frac{d^{2} \sigma}{d \Omega d E^{\prime}}=r_{0}^{2} \frac{k_{f}}{k_{i}} S(\mathbf{Q}, \omega),
$$

where $k_{i}$ and $k_{f}$ are the initial and final neutron wave numbers, $r_{0}$ is the classical electron radius, and $\mathrm{S}(\mathbf{Q}, \omega)$ is the Fourier transform of the spin-spin correlation function:

$$
S(\mathbf{Q}, \omega)=\frac{1}{2 \pi \hbar} \sum_{\alpha \beta}\left(\delta_{\alpha \beta}-\hat{Q}_{\alpha} \hat{Q}_{\beta}\right) \int_{-\infty}^{\infty} d t e^{-i \omega t}\left\langle\hat{S}_{Q}^{\alpha} \hat{S}_{-Q}^{\beta}(t)\right\rangle .
$$

In the Heisenberg model, the spin amplitudes are written as the Fourier transform of the spin density

$$
\hat{S}_{\mathbf{Q}}^{\alpha}(t)=\sum_{l i} f_{i}(Q) e^{-i \mathbf{Q} \cdot\left(\mathbf{l}+\mathbf{d}_{i}\right)} \hat{S}_{l i}^{\alpha},
$$

for magnetic ions at sites $\mathbf{d}_{i}$. The amplitude prefactor $f_{i}(Q)$ depends on the Lande $g$-factor, the magnetic form factor, $F_{i}(Q)$, and the Debye-Waller factor, $W_{i}(Q)$, for the magnetic ion

$$
f_{i}(Q)=\frac{1}{2} g_{i} F_{i}(\mathrm{Q}) e^{-W_{i}(\mathrm{Q})} .
$$

By expanding the local spin deviation in terms of plane waves

$$
\hat{S}_{l i}^{\alpha}(t)=\frac{1}{N} \sum_{\mathbf{q}} e^{i \mathbf{q} \cdot 1} \hat{S}_{\mathbf{q}, i}^{\alpha}(t)
$$

the above correlation function becomes

$$
\begin{aligned}
\left\langle\hat{S}_{\mathbf{Q}}^{\alpha} \hat{S}_{-\mathbf{Q}}^{\beta}(t)\right\rangle= & \sum_{i j} f_{i}(Q) f_{j}(Q) e^{i \mathbf{Q} \cdot\left(\mathbf{d}_{j}-\mathbf{d}_{i}\right)} \sum_{\mathbf{q}} \delta(\mathbf{Q}-\mathbf{q}-\tau) \\
& \times\left\langle\hat{S}_{\mathbf{q}, i}^{\alpha} \hat{S}_{-\mathbf{q}, j}^{\beta}(t)\right\rangle .
\end{aligned}
$$

The evaluation of the thermal averages of the spin-spin correlation functions for spin wave deviations of the type $S^{+} S^{-}$ are performed for each branch labeled $n$

$$
\left\langle S_{\mathbf{q}, i}^{+} S_{\mathbf{q}, j}^{-}(t)\right\rangle_{n}=\frac{\sigma_{i} \sigma_{j}}{2 N} \sqrt{S_{i} S_{j}} T_{n i} T_{n j}^{*}\left[n_{\mathbf{q}, n} \exp \left(-i \omega_{n}(\mathbf{q}) t\right)\right],
$$


TABLE III. Iron positions and spins for magnetite in the primitive cell of the cubic $\mathrm{Fd} \overline{3} \mathrm{~m}$ structure with rhombohedral direct lattice vectors: $\left(a_{c} / 2, a_{c} / 2,0\right),\left(a_{c} / 2,0, a_{c} / 2\right),\left(0, a_{c} / 2, a_{c} / 2\right)$.

\begin{tabular}{cccccc}
\hline \hline$i$ & Fe position & $\sigma_{i} S_{i}\left(\mu_{B}\right)$ & $d_{x}$ & $d_{y}$ & $d_{z}$ \\
\hline 1 & $\mathrm{~A}, \overline{4} 3 \mathrm{~m}$ & -2.5 & 0 & 0 & 0 \\
2 & $\mathrm{~A}, \overline{4} 3 \mathrm{~m}$ & -2.5 & $1 / 4$ & $1 / 4$ & $1 / 4$ \\
3 & $\mathrm{~B}, \overline{3} \mathrm{~m}$ & 2.25 & $-1 / 8$ & $-3 / 8$ & $-1 / 8$ \\
4 & $\mathrm{~B}, \overline{3} \mathrm{~m}$ & 2.25 & $-3 / 8$ & $-1 / 8$ & $-1 / 8$ \\
5 & $\mathrm{~B}, \overline{3} \mathrm{~m}$ & 2.25 & $-1 / 8$ & $-1 / 8$ & $-3 / 8$ \\
6 & $\mathrm{~B}, \overline{3} \mathrm{~m}$ & 2.25 & $-3 / 8$ & $-3 / 8$ & $-3 / 8$ \\
\hline \hline
\end{tabular}

$$
\left\langle S_{-\mathbf{q}, i}^{-} S_{-\mathbf{q}, j}^{+}(t)\right\rangle_{n}=\frac{\sigma_{i} \sigma_{j}}{2 N} \sqrt{S_{i} S_{j}} T_{n i} T_{n j}^{*}\left[\left(n_{-\mathbf{q}, n}+1\right) \exp \left(i \omega_{n}(-\mathbf{q}) t\right)\right],
$$

where $n_{\mathbf{q} n}$ is the Bose occupation factor and $T_{n i}$ is the contribution of the $i$ th atom to the spin wave eigenvector of branch $n$.

For collinear spins aligned (anti) parallel to the $z$ axis, the spin wave cross section can then be written $(\mathbf{q}=-\mathbf{q})$ as:

$$
\begin{aligned}
S(\mathbf{Q}, \omega)= & \left(1+\hat{Q}_{z}^{2}\right) \sum_{\mathbf{q}, n}\left|\sum_{i} f_{i}(Q) \sigma_{i} \sqrt{S_{i}} T_{n i} e^{-i \mathbf{Q} \cdot \mathbf{d}_{i}}\right|^{2} \\
& \times \delta(\mathbf{Q}-\mathbf{q}-\boldsymbol{\tau})\left\{n_{\mathbf{q}, n} \delta\left[\omega+\omega_{n}(\mathbf{q})\right]\right. \\
& \left.+\left(n_{\mathbf{q}, n}+1\right) \delta\left[\omega-\omega_{n}(\mathbf{q})\right]\right\} .
\end{aligned}
$$

For comparison to the measured intensities, the correlation function above is convoluted with the experimental resolution function.

$$
I\left(\mathbf{Q}_{0}, \omega_{0}\right)=\int S(\mathbf{Q}, \omega) R\left(\mathbf{Q}-\mathbf{Q}_{0}, \omega-\omega_{0}\right) d^{3} \mathbf{Q} d \omega .
$$

The resolution function, $R(\mathbf{Q}, \omega)$, is calculated from the experimental configuration parameters in Table II and other information, such as the crystal mosaic spreads, using the Cooper-Nathans formalism. ${ }^{27}$ Convolutions of Heisenberg model results and other analytical approximations to the dispersion relations were performed with the RESLIB program. ${ }^{28}$

\section{E. Spin wave calculations for magnetite in cubic spinel phase}

At high temperatures, magnetite has the cubic spinel structure in the $\mathrm{Fd} \overline{3} \mathrm{~m}$ space group. The iron atoms in the tetrahedral interstices (A sites) have a valence of $\mathrm{Fe}^{3+}$. Iron atoms in octahedral interstices (B sites) have an average valence of $\mathrm{Fe}^{2.5+}$. The positions and spins of the iron atoms in the cubic primitive cell are given in Table III. Table IV lists the pair-wise Heisenberg exchange values used for cubic magnetite. ${ }^{10}$ Figure 8 shows the results of a numerical calculation of the spin waves in magnetite along various symmetry directions using the model parameters in Tables III and IV.
TABLE IV. Nearest-neighbor pair-wise superexchange values used for magnetite in the cubic phase (from Ref. 10).

\begin{tabular}{cc}
\hline \hline Pair, $(0 i ; l j)$ & $J(0 i: l j)(\mathrm{meV})$ \\
\hline $\mathrm{AB},(01 ; 03)$ & -4.8 \\
$\mathrm{AA},(01 ; 02)$ & 0.0 \\
$\mathrm{BB},(03 ; 04)$ & 0.48 \\
\hline \hline
\end{tabular}

Using the model results, the mode eigenvectors can be analyzed to sort out the spin deviations in each branch. Table $\mathrm{V}$ shows the spin deviation amplitude at each atomic site for each mode at $\mathbf{q}=0$. The acoustic mode $\left(\omega_{1}\right)$ has equal amplitude spin precession on each site. The strongly dispersing optic mode has a similar eigenvector to the acoustic mode, but has a larger spin deviation on the A site in response to the large internal molecular field. The flat optic branches between $70-80 \mathrm{meV}$ are propagating on the B sublattice only. Likewise, the flat $130 \mathrm{meV}$ branch propagates on the A sublattice.

\section{F. Resolution function convolutions}

In order to achieve a complete understanding of the spin wave dispersion and splitting below $T_{V}$, careful studies of the effects of instrumental resolution must be made. This is especially true considering that the spin wave dispersion is steep, and peak shapes can have long and asymmetric tails due to resolution effects. The resolution must be understood before statements can be made about lifetimes of the excitations obtained from intrinsic peakwidths. Finally, since a fairly accurate model exists for the high-temperature spin

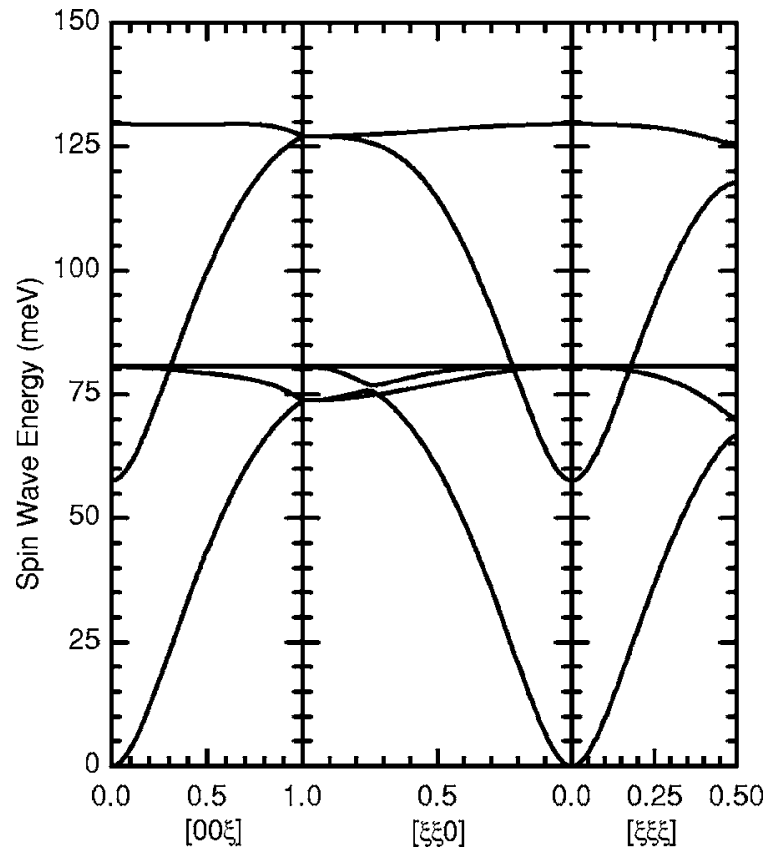

FIG. 8. Spin wave dispersion of cubic magnetite along the principal symmetry directions as calculated from a Heisenberg model. (Heisenberg parameters were obtained from Ref. 10.) 
TABLE V. The spin wave eigenvectors at $\mathbf{q}=0$ from the Heisenberg model in the cubic phase of magnetite.

\begin{tabular}{ccccccc}
\hline \hline & $\begin{array}{c}T_{1 i} \\
\left(\omega_{1}=0\right)\end{array}$ & $\begin{array}{c}T_{2 i} \\
\left(\omega_{2}=58 \mathrm{meV}\right)\end{array}$ & $\begin{array}{c}T_{3 i} \\
\left(\omega_{3}=81 \mathrm{meV}\right)\end{array}$ & $\begin{array}{c}T_{4 i} \\
\left(\omega_{4}=81 \mathrm{meV}\right)\end{array}$ & $\begin{array}{c}T_{5 i} \\
\left(\omega_{5}=81 \mathrm{meV}\right)\end{array}$ & $\begin{array}{c}T_{6 i} \\
\left(\omega_{6}=130 \mathrm{meV}\right)\end{array}$ \\
\hline 1 & 0.378 & 0.567 & 0 & 0 & 0 & -0.707 \\
2 & 0.378 & 0.567 & 0 & 0 & 0 & 0.707 \\
3 & 0.423 & 0.315 & -0.471 & 0.613 & -0.364 & 0 \\
4 & 0.423 & 0.315 & -0.471 & -0.613 & 0.364 & 0 \\
5 & 0.423 & 0.282 & 0.527 & 0.352 & 0.605 & 0 \\
6 & 0.423 & 0.282 & 0.527 & -0.352 & -0.605 & 0 \\
\hline \hline
\end{tabular}

wave spectrum, we can use the convolutions as a guide to search for phonon excitations that may be overlapping the spin waves.

We begin with the data measured in the cubic phase above the Verwey transition and verify that the Heisenberg model from Ref. 10 agrees with the present data. Using the parameters in Tables III and IV, the acoustic spin wave dispersion along [001], as shown in Fig. 8, is plotted again in Fig. 9(a). Also shown in Fig. 9(a) are the resolution ellipsoids at various $(\mathbf{Q}, \omega)$ points along the dispersion for different instrument configurations. Figures 9(b)-9(f) show various measured constant- $\mathbf{Q}$ energy cuts through the spin waves plotted along with calculations of the resolution convoluted Heisenberg model cross sections. The general agreement validates the original Heisenberg model.

In order to expedite detailed fits to the spin wave data and extract other parameters, such as peak widths and the position of nearby phonon excitations, an analytical expression for the spin wave dispersion has been developed. The analytical expression is based on a sigmoidal function that reproduces the acoustic spin wave dispersion in the cubic phase of magnetite,

$$
\hbar \omega(\mathbf{q})=D q_{0}^{2}\left[1-\frac{q_{0}^{2}}{|\mathbf{q}|^{2}+q_{0}^{2}}\right],
$$

where $D$ is the spin wave stiffness and $q_{0}$ is the curve shape parameter. We make the assumption (verified by Heisenberg model calculations) that the dispersion is isotropic and has the correct $D q^{2}$ limit as $|\mathbf{q}|$ goes to zero. This function is a very good approximation to the dispersion of the acoustic mode as calculated from the Heisenberg model (see Fig. 10). We assume that the spin wave peaks have some intrinsic Lorentzian broadening. Due to the steepness of the dispersion and the relatively broad resolution function, spin waves at many different $\mathbf{q}$ values are folded into the convolved cross section. Therefore, the mode intensities must be well defined for a good fit. By analysis of the Heisenberg model calculations, the following function reproduces the nearly isotropic spin wave structure factors in the $(0,0,4)$ Brillouin zone,

$$
S(\mathbf{Q}, \omega) \approx I_{0} f^{2}(Q) \cos \left(\frac{q a}{8}\right) \frac{1}{1-\exp (-\hbar \omega / k T)} .
$$

Using this function, we are able to fit the high-temperature energy scans. During these fits, the parameter $q_{0}$ was held fixed and the spin wave stiffness, width, and intensity were varied. With $q_{0} \approx 0.546 \mathrm{rlu}$, typically $D \approx 330 \mathrm{meV} \mathrm{rlu}^{-2}$ and Lorentzian peakwidths of $0.5-2 \mathrm{meV}$ are found. For scans where both spin wave and phonon excitations are present, these were fit simultaneously. Figure 11(a) shows the measured dispersion obtained from fits to all of the data above $T_{V}$.

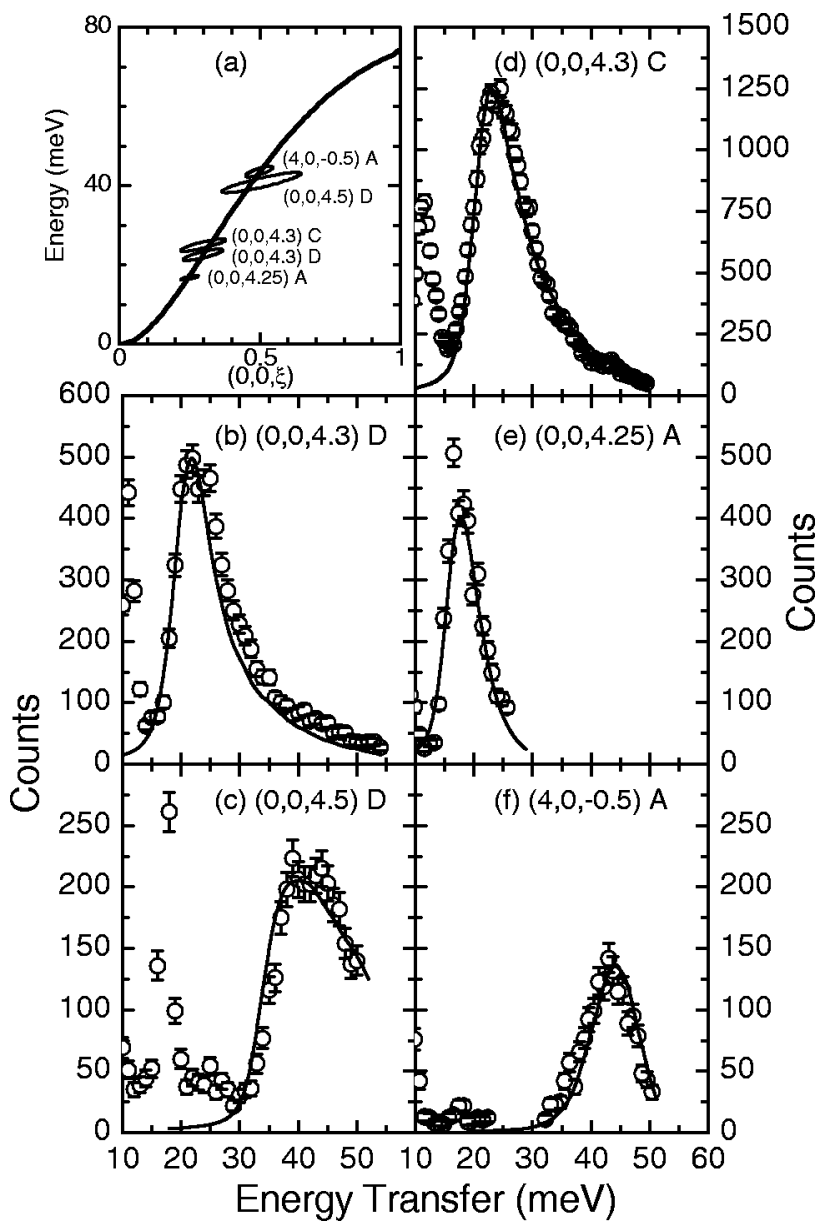

FIG. 9. (a) The acoustic spin wave dispersion of magnetite along [001] and above $T_{V}$ as calculated from the Heisenberg model of Ref. 10 and projections of the resolution ellipsoids for the various experimental configurations. (b)-(f) show various resolution convoluted cross sections obtained from the Heisenberg model as compared to the measured data for the experimental configurations annotated on the figures. 


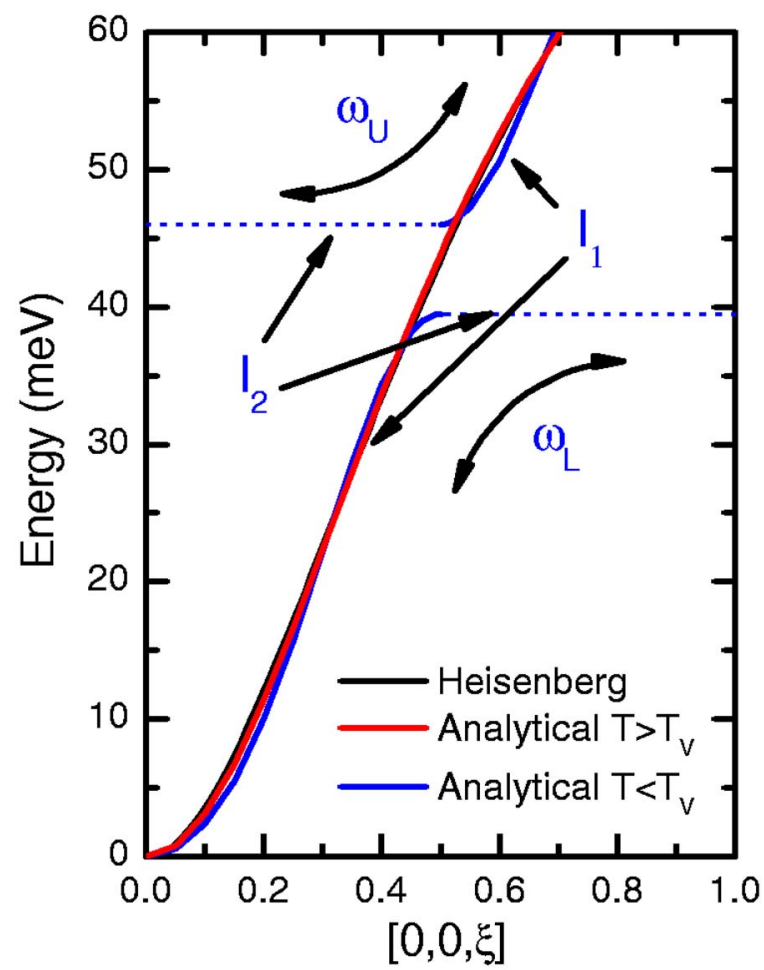

FIG. 10. (Color online) Analytical curves for the acoustic spin wave dispersion along [001] are compared to the Heisenberg model (black). The red curve is calculated from the high-temperature formula given in Eq. (20). The blue curves are the two lowtemperature branches with $\omega_{L}\left(\omega_{U}\right)$ the lower (upper) branch as calculated in Eq. (22). The continuation of these branches is presumed to be flat and is shown by dotted lines. The dispersive parts of $\omega_{L}$ and $\omega_{U}$ are primarily cubic modes whose strong intensity is governed by the fitting parameter $I_{1}$ (solid blue curve) while the weak and flat parts are governed by $I_{2}$ (dotted blue curve).

The presence of the spin wave splitting below $T_{V}$ is not captured by the simple Heisenberg Hamiltonian presented in Sec. III C. In order to fit the data, we have developed other analytical functions to represent the two (split) branches of the low-temperature acoustic spin wave dispersion. The dispersion relations of the split branches are labeled $\mathrm{U}$, for the upper branch, and $L$ for the lower branch. When $q<1 / 2$, the dispersion relations are fit to the functions

$$
\begin{aligned}
& \hbar \omega_{L}(\mathbf{q})=E_{1} \sin ^{2}(q a / 2)-E_{1}^{\prime} \sin ^{2}(q a), \\
& \hbar \omega_{U}(\mathbf{q})=E_{1}+\Delta .
\end{aligned}
$$

For $q>1 / 2$, the dispersions of the two branches are

$$
\begin{gathered}
\hbar \omega_{L}(\mathbf{q})=E_{1}, \\
\hbar \omega_{U}(\mathbf{q})=E_{2}-\left(E_{2}-E_{1}-\Delta\right) \sin ^{2}(q a / 2)+E_{2}^{\prime} \sin ^{2}(q a) .
\end{gathered}
$$

For these functions, $\Delta$ is the energy splitting at $\mathbf{q}=(0,0,1 / 2)$, giving two modes with energies $E_{1}$ and $E_{1}+\Delta$ at this wavevector. The additional parameters; $E_{1}^{\prime}, E_{2}$, and $E_{2}^{\prime}$ are used to fit the overall dispersion shape. Typical

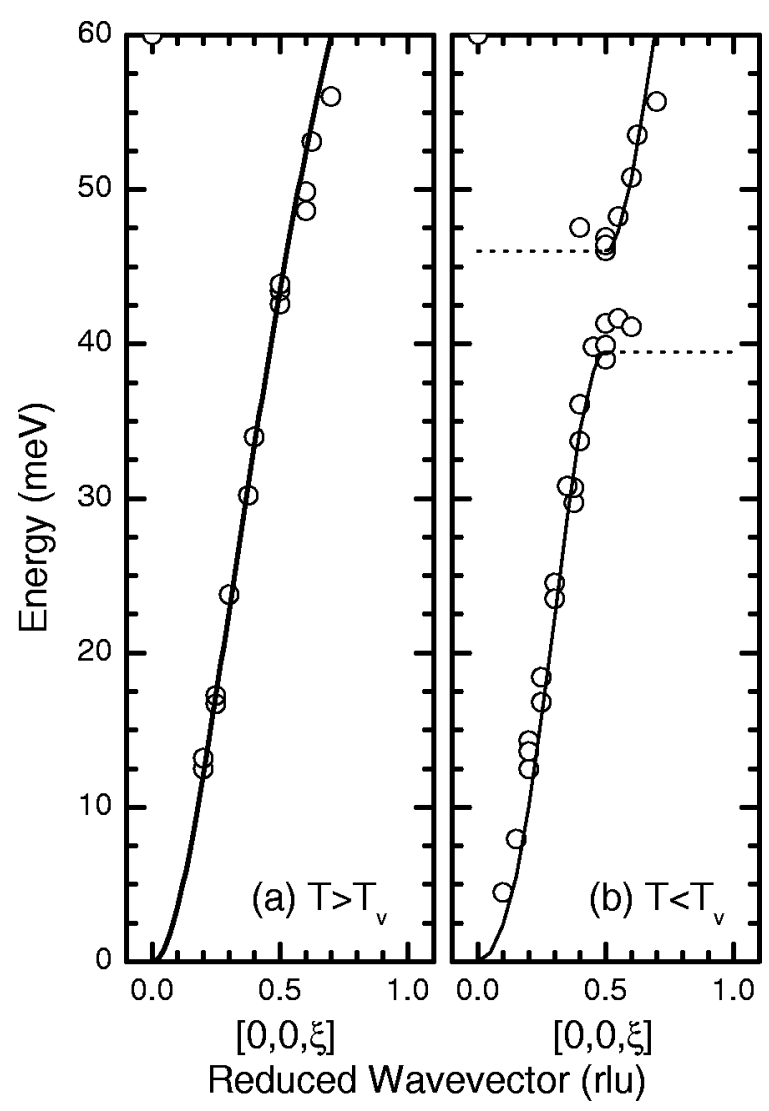

FIG. 11. The acoustic spin wave dispersion of magnetite along [001] as obtained from fits to the resolution folded analytical dispersion relations in various experimental configurations (a) above $T_{V}$, and (b) below $T_{V}$. Empty circles are the fitted energies of the spin wave modes. The solid lines are the analytical dispersion relations for typical values of the fitting parameters. The dotted line in (b) indicates a possible continuation of the dispersion of the upper and lower branches.

values of the dispersion parameters determined from fitting are: $E_{1}=40 \mathrm{meV}, E_{1}^{\prime}=3 \mathrm{meV}, E_{2}=73 \mathrm{meV}$, and $\Delta=7 \mathrm{meV}$. The two branches $\omega_{L}$ and $\omega_{\mathrm{U}}$ are plotted in Fig. 10 for typical values of the fitting parameters.

At low temperatures, we used the following functional form for the spin wave mode intensities of branches $\mathrm{L}$ and $\mathrm{U}$ when $0<q<1 / 2$;

$$
S_{L}\left(\mathbf{Q}, \omega_{L}\right) \approx I_{1} f^{2}(Q) \cos \left(\frac{q a}{8}\right) \frac{1}{1-\exp \left(\hbar \omega_{L} / k T\right)},
$$

$$
S_{U}\left(\mathbf{Q}, \omega_{U}\right)=I_{2} f^{2}(Q) \cos \left(\frac{a}{16}\right) \frac{1}{1-\exp \left(\hbar \omega_{U} / k T\right)}
$$

For $1 / 2<q<1$,

$$
S_{L}\left(\mathbf{Q}, \omega_{L}\right) \approx I_{2} f^{2}(Q) \cos \left(\frac{a}{16}\right) \frac{1}{1-\exp \left(\hbar \omega_{L} / k T\right)},
$$




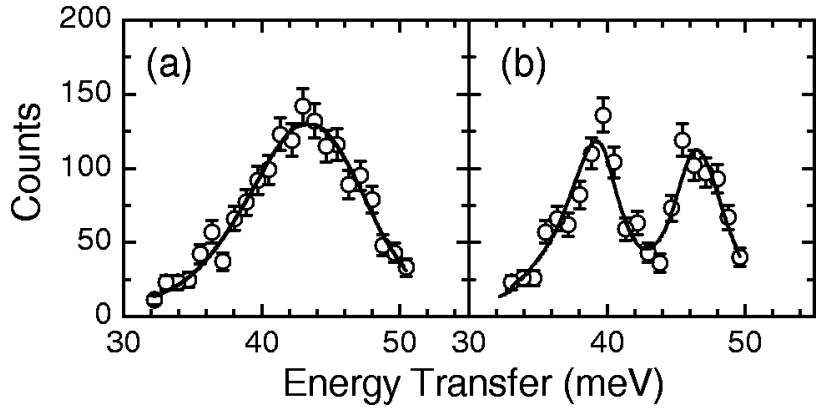

FIG. 12. Resolution folded fits to the spin wave excitation at $(4,0,-1 / 2)$ as measured in experimental configuration $\mathrm{A}$ at (a) $T=130 \mathrm{~K}$ and (b) $T=115 \mathrm{~K}$. Open circles are the experimental data and solid lines are resolution folded fits. The fitting gives an intrinsic spin wave width of $\sim 1.5 \mathrm{meV}$ both above and below $T_{V}$. Thus, the apparent narrowing of the spin wave modes below $T_{V}$ is a resolution effect due to the flattening of the dispersion near the gap.

$$
S_{U}\left(\mathbf{Q}, \omega_{U}\right) \approx I_{1} f^{2}(Q) \cos \left(\frac{q a}{8}\right) \frac{1}{1-\exp \left(-\hbar \omega_{U} / k T\right)} .
$$

The parameter $I_{1}$ represents the intensity of the main steeply dispersing and gapped spin wave branch (consisting of the $\mathrm{L}$ branch when $q<1 / 2$ and the $\mathrm{U}$ branch when $q>1 / 2$ ) while $I_{2}$ is the intensity of any weak and relatively dispersionless extra branch that may arise from symmetry lowering. The intensity functions used in the fitting are associated with the branches as shown Fig. 10.

At $\mathbf{q}=(0,0,1 / 2)$, the resolution folded fits are shown in Fig. 12 both above and below $T_{V}$. The rather broad spin wave above $T_{V}$ becomes two relatively narrow excitations below $T_{V}$. Based on our resolution convolved fits, the narrowing of the spin waves below $T_{V}$ is a resolution effect and not a narrowing of the intrinsic width of the peaks. The shape of the low-temperature dispersion near $(0,0,1 / 2)$ flattens out near the gap, no longer being steeply dispersive, giving rise to resolution narrowing effect. The intrinsic width of the spin wave peaks above and below $T_{V}$ is the same, $\sim 1.5 \mathrm{meV}$. Fits at other values of $\mathbf{q}$ and for several experimental configurations are summarized in Fig. 11.

Constant-Q scans on either side of $\mathbf{q}=(0,0,1 / 2)$ show multiple peaks that appear to arise from an additional weak and flatly dispersing excitation branch. Such multiple peaks can be seen in Fig. 4 in the range of $\mathbf{Q}=(0,0,4.35)$ to $(0,0,4.55)$. However, convolutions of the analytical form in Eqs. (22) and (23) show that the extra peaks arise from the gapped spin waves at $\mathbf{q}=(0,0,1 / 2)$, and not from any additional modes away from $(0,0,1 / 2)$. In fact, the parameter $I_{2}$ can be set to zero without seriously affecting the resolution folded peak shapes. An example of extra peaks appearing due to resolution is observed in constant- $\mathbf{Q}$ energy cuts of the spin wave at $\mathbf{Q}=(0,0,4.4)$ in Fig. 13. Thus, if the splitting arises from symmetry lowering or mixing with another excitation, any newly appearing branches are extremely weak. This is indicated in Fig. 11, where the dashed horizontal dispersion lines are one possible continuation of the upper and lower spin wave branches.

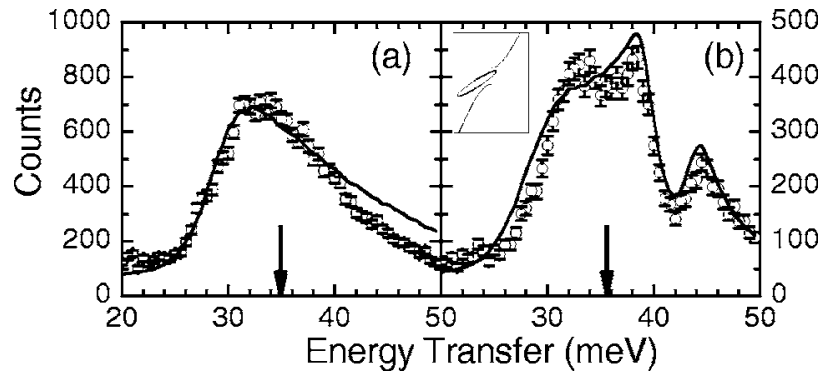

FIG. 13. Constant-Q energy cuts of the spin wave at $\mathbf{Q}=(0,0,4.4)$ in configuration $\mathrm{C}$ at (a) $T=300 \mathrm{~K}$, and (b) $T=115 \mathrm{~K}$. Dots are the measured data and lines are the result of convolution of the analytical expressions for $S(\mathbf{Q}, \omega)$ with the resolution function. Arrows point to the fitted spin wave energy for each model. While (a) contains only a single excitation, (b) appears to contain three excitations at $\mathbf{Q}=(0,0,4.4)$. However, the resolution calculations were performed with the parameter $I_{2}=0$ in (b) meaning that only one branch exists in the model at $(0,0,4.4)$. The two higher-energy peaks at $38 \mathrm{meV}$ and $44 \mathrm{meV}$ are the split modes at $(0,0,4.5)$ which are picked up by the tail of the resolution function, as shown schematically in the inset. The dispersion of any weak extra branch is difficult to pick up due to the combination of steep dispersion and coarse energy resolution.

The combination of steep dispersion, coarse resolution, and fairly large values of neutron incident energy requires detailed knowledge of the full $S(\mathbf{Q}, \omega)$ and the resolution function over large regions of $(\mathbf{Q}, \omega)$ space. In addition, at the limit of large incident energies, the incident collimation is effectively reduced (and energy resolution improved) because the monochromator is viewed to be smaller at shallow scattering angles. In the coarsest resolution measurements presented here (configuration D, for example), we are sometimes able to achieve only marginal fits within the CooperNathans formalism. Higher-resolution measurements are required to determine the full dispersion of the two branches below $T_{V}$. In general, the fits above and below $T_{V}$ reflect the fact that the dispersion is only modified close to $\mathbf{q}=(0,0,1 / 2)$. At values of $\mathbf{q}$ some distance away from $(0,0,1 / 2)$, the dispersion is the same as that above $T_{V}$. We also found no substantive difference in the peakwidths above and below $T_{V}$.

\section{ANALYSIS OF SPIN WAVES IN MAGNETITE BELOW $T_{v}$}

There are several possible origins of the spin wave splitting in magnetite below the Verwey temperature. A plausible origin of the splitting is due to the lowering of crystallographic symmetry in the Verwey phase. This symmetry lowering causes small changes in the superexchange intergrals due to distortions in the metal-oxygen bond lengths and bond angles. If charge ordering is present, then this also modifies the superexchange due to variations in orbital occupancy. In this section, both of these possiblities are considered by developing detailed Heisenberg models in the low symmetry state. 
TABLE VI. Iron and oxygen positions in magnetite below the Verwey transition in the Pmca space group (from Ref. 12).

\begin{tabular}{ccccc}
\hline \hline Atom & Site & \multicolumn{1}{c}{$d_{x}$} & \multicolumn{1}{c}{$d_{y}$} & \multicolumn{1}{c}{$d_{z}$} \\
\hline A1 & $4(\mathrm{~d}), \mathrm{m}$ & 0.25 & 0.0049 & 0.0635 \\
A2 & $4(\mathrm{~d}), \mathrm{m}$ & 0.25 & 0.5067 & 0.1887 \\
B1 & $4(\mathrm{~b}), \overline{1}$ & 0 & 0.5 & 0 \\
B2 & $4(\mathrm{c}), 2$ & 0 & 0.0099 & 0.25 \\
B3 & $4(\mathrm{~d}), \mathrm{m}$ & 0.25 & 0.2643 & 0.3789 \\
B4 & $4(\mathrm{~d}), \mathrm{m}$ & 0.25 & 0.7549 & 0.3746 \\
O1 & $4(\mathrm{~d}), \mathrm{m}$ & 0.25 & 0.2630 & -0.0027 \\
O2 & $4(\mathrm{~d}), \mathrm{m}$ & 0.25 & 0.7477 & -0.0009 \\
O3 & $4(\mathrm{~d}), \mathrm{m}$ & 0.25 & 0.2461 & 0.2540 \\
O4 & $4(\mathrm{~d}), \mathrm{m}$ & 0.25 & 0.7696 & 0.2527 \\
O5 & $8(\mathrm{e}), 1$ & -0.0116 & 0.0089 & 0.1295 \\
O6 & 8(e), 1 & -0.0067 & 0.5050 & 0.1244 \\
\hline \hline
\end{tabular}

A. Dependence of the spin wave spectrum on small crystalline distortions

Even in the absence of charge ordering on the B sites, the detailed pair-wise superexchange values depend on the $\mathrm{Fe}-\mathrm{O}-\mathrm{Fe}$ distances and bond angles and will be modified by the small crystalline distortions. To develop a Heisenberg model in the low symmetry Verwey phase, the orthorhombic space group is used. While the correct space group is likely the monoclinic $\mathrm{Cc}$ group, it has been shown that all but the three weakest superlattice reflections can be described by the orthorhombic group Pmca. ${ }^{3}$ The Pmca space group does include the $(0,0,1 / 2)$-type superlattice reflections (cell doubling) which are of importance in the splitting of the spin wave dispersion. The Pmca unit cell of magnetite is indexed relative to the original cubic cell according to the scaling $a / \sqrt{2} \times a / \sqrt{2} \times 2 a$. Within this space group, magnetite contains two unique $\mathrm{A}$ sites and four unique B sites, each with a multiplicity of four giving a total of 24 magnetic iron sites. The collinear moment directions point along the $c$ axis in the Pmca space group. Table VI shows the unique Fe and $\mathrm{O}$ sites in the Pcma structure.

The pair-wise exchange values will vary throughout the larger cell due to atomic distortions in the orthorhombic structure. The dependence of the superexchange on bond distances and angles is a difficult theoretical problem, and there is no simple quantitative relationship for its dependence. However, in this instance, we only require knowledge of the small corrections to the superexchange relative to the experimentally determined cubic values. Exact-diagonalization calculations and perturbation theory ${ }^{29}$ have shown that for the transition metal oxides, the superexchange is approximately proportional to

$$
J_{\mathrm{MN}} \propto t_{\mathrm{MO}}^{2} t_{\mathrm{NO}}^{2} \cos ^{2} \theta_{\mathrm{MON}}
$$

where $\mathrm{M}, \mathrm{N}=A$ or $B$ and $t_{\mathrm{MO}}$ is the metal-oxygen transfer integral. The $p d$ transfer integrals depend sensitively on the metal-oxygen distance $\left(d_{\mathrm{MO}}\right)$ as $t_{\mathrm{MO}} \propto d_{\mathrm{MO}}^{-7 / 2} \cdot{ }^{30}$ In the limit of
TABLE VII. Local variations in the iron-oxygen bond distances and bond angles and the corresponding variation of the superexchange in the Pmca structure. The cubic values are $d_{\mathrm{AO}}^{c}=1.876 \AA$, $d_{\mathrm{BO}}^{c}=2.066 \AA, \theta_{\mathrm{AOB}}^{c}=123.95^{\circ}$.

\begin{tabular}{cccc}
\hline \hline$d_{\mathrm{AO}}(\AA)$ & $d_{\mathrm{BO}}(\AA)$ & $\theta_{\mathrm{AOB}}(\mathrm{deg})$ & $J_{\mathrm{AB}} / J_{\mathrm{AB}}^{c}$ \\
\hline 1.893 & 2.045 & 122.93 & 0.958 \\
1.908 & 2.025 & 123.58 & 1.003 \\
1.908 & 2.068 & 121.89 & 0.768 \\
1.893 & 2.063 & 123.02 & 0.900 \\
1.908 & 2.078 & 121.58 & 0.719 \\
1.871 & 2.012 & 127.34 & 1.353 \\
1.871 & 2.089 & 124.75 & 0.987 \\
1.894 & 2.049 & 122.12 & 0.895 \\
1.896 & 2.042 & 122.74 & 0.945 \\
1.867 & 2.070 & 124.09 & 1.023 \\
1.867 & 2.032 & 126.82 & 1.298 \\
1.894 & 2.058 & 123.92 & 0.956 \\
1.867 & 2.090 & 124.24 & 0.968 \\
1.896 & 2.100 & 122.39 & 0.730 \\
\hline \hline
\end{tabular}

small atomic displacements, we can relate the superexchange of a given $\mathrm{M}-\mathrm{N}$ pair to the cubic value as

$$
\frac{J_{\mathrm{MN}}}{J_{\mathrm{MN}}^{c}} \approx\left(1-\frac{7(\Delta d)_{\mathrm{MO}}}{d_{\mathrm{MO}}^{c}}-\frac{7(\Delta d)_{\mathrm{NO}}}{d_{\mathrm{NO}}^{c}}-2(\Delta \theta)_{\mathrm{MON}} \tan \theta_{\mathrm{MON}}^{c}\right) \text {, }
$$

where $J_{\mathrm{MN}}^{c}$ is the exchange value in the undistorted cubic structure and $\Delta d(\Delta \theta)$ is the bond length (bond angle) deviation from the corresponding cubic value $d^{c}\left(\theta^{c}\right)$. Using this prescription for modifying the superxchange values, we tabulated all of the unique $\mathrm{AB}$ exchange paths in the Pmca structure. There are 14 distinct $\mathrm{Fe}-\mathrm{O}(\mathrm{AO}, \mathrm{BO})$ pair distances. The AOB bond angles, pair distances, and the corresponding ratio of $J_{\mathrm{AB}} / J_{\mathrm{AB}}^{c}$ are given in Table VII. Table VII reveals that some pairs can have their superexchange modified by as much as $30 \%$ from the cubic value despite the rather small crystalline distortions from the cubic positions. Using the modified superexchange values, the spin wave dispersion was calculated along the cubic [001] direction and is shown in Fig. 14(a). There are obviously many more branches in the Pmca model (24 total), but most of these branches arise from folding the cubic branches into the smaller Pmca Brillouin zone. Figure 15 shows that the neutron intensities calculated around the (004) zone from this model show only the two main dispersive cubic branches, all other folded-in branches have very low intensity due to extremely weak structure factors originating from small crystalline distortions. However, the spin wave calculations do show some effects beyond zone folding that depend on the varying exchange values. For example, a reasonably large gap exists in the dispersing optic mode at $(0,0,1 / 2)$ and the degeneracy of optic modes along the face of the $\mathrm{Fd} \overline{3} \mathrm{~m}$ Brillouin zone are lifted as expected from symmetry lowering. 


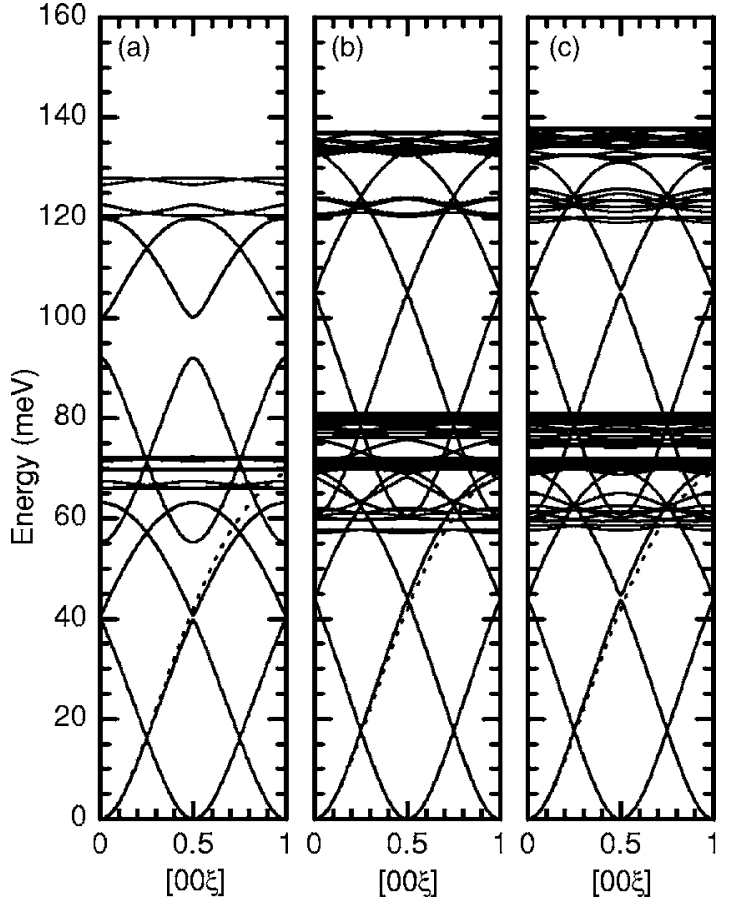

FIG. 14. Low-temperature spin wave dispersion for magnetite along the original cubic [001] direction as calculated for a Heisenberg model with superexchange interactions modified by (a) atomic distortions in the Pmca structure, (b) charge ordering obeying the Anderson condition and, (c) charge ordering violating the Anderson condition. In each figure, the dashed line corresponds to the original high-temperature acoustic spin wave branch.

However, the symmetry lowering model introduces no significant gap in the acoustic spin wave at $(0,0,1 / 2)$.

\section{B. Dependence of the spin wave spectrum on charge ordering}

As discussed above, charge order on the B sites will also influence the pair-wise superexchange paths. We must now consider $\mathrm{AB}^{\prime}$ and $\mathrm{AB}^{\prime \prime}$ superexchange paths, where $B^{\prime}$ is an $\mathrm{Fe}^{3+}$ ion $(S=5 / 2)$ and $B^{\prime \prime}$ is an $\mathrm{Fe}^{2+}$ ion $(S=2)$. In order to investigate the effect of charge order on the spin waves, we must know how the $J_{\mathrm{AB}^{\prime}}$ and $J_{\mathrm{AB}^{\prime \prime}}$ superexchange values differ from the average superexchange $J_{\mathrm{AB}}$ and we must also know the actual charge ordering pattern. At this point, we ignore the small atomic displacements treated in the previous section and use the cubic atomic positions. The superexchange is ${ }^{19,31}$

$$
J_{i j}=\frac{1}{4 S_{i} S_{j}} \sum_{k} \frac{2 b_{k}^{2}}{U_{k}}
$$

for all possible superexchange paths $k$, where $U_{k}$ is the effective Coulomb repulsion parameter and $b_{k} \propto\left(t_{\mathrm{AO}}^{i} t_{\mathrm{BO}}^{j}\right)_{k}$. Given that the spinel AOB angle is $125^{\circ}$, there are hundreds of possible superexchange paths due to the nonorthogonality of the $3 d$ states on the $\mathrm{A}$ and $\mathrm{B}$ sites. If we assume a $180^{\circ}$ bond angle, we only need to consider superexchange paths due to $\sigma$-bonds (through the $e_{g}$ orbitals) and $\pi$-bonds (through the $t_{2 g}$ orbitals). This approximation will allow a

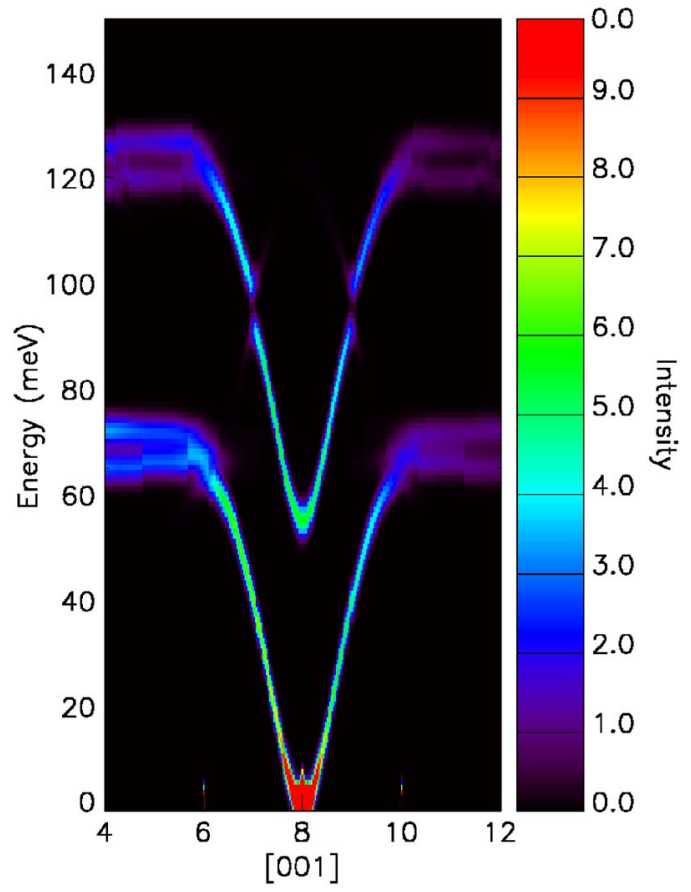

FIG. 15. (Color online) Neutron intensities along [001] in the Pmca structure with locally varied superexchange due to atomic distortions. Despite the large number of branches which are folded into the smaller Pmca zone, only the main cubic branches show appreciable intensity due to the small atomic distortions in the Verwey phase.

rough estimation of the dependence of the superexchange on orbital occupancy without detailed analysis using the SlaterKoster integrals and local electron configurational energies. For $\mathrm{AB}^{\prime}$, there is then one $\sigma$-bond and two $\pi$-bonds. For $\mathrm{AB}^{\prime \prime}$, there is again one $\sigma$-bond since the B site $e_{g}$ levels are unaffected, but the average number of $\pi$-bonds decreases to $4 / 3$ due to the presence of an extra electron in the octahedral $t_{2 g}$ orbital (there are three orbitals that the extra $t_{2 g}$ electron can occupy, $x z, y z, x y$ and a total of four superexchange paths amongst the three possible orbital occupations). Given this simplification, the effect of superexchange on charge ordering is

$$
J_{\mathrm{AB}^{\prime}}=-\frac{1}{25}\left(\frac{2 b_{\sigma}^{2}}{U^{\prime}}+\frac{4 b_{\pi}^{2}}{U^{\prime}}\right), \quad J_{\mathrm{AB}^{\prime \prime}}=-\frac{1}{20}\left(\frac{2 b_{\sigma}^{2}}{U^{\prime \prime}}+\frac{4}{3} \frac{2 b_{\pi}^{2}}{U^{\prime \prime}}\right) .
$$

It is estimated that $U^{\prime} \approx 10 \mathrm{eV}, \quad U^{\prime \prime} \approx 8 \mathrm{eV}$, and $b_{\pi}^{2} \approx 0.1 b_{\sigma}^{2}, 32,33$ we then obtain the following ratios; $2 J_{\mathrm{AB}^{\prime}} /\left(J_{\mathrm{AB}^{\prime}}+J_{\mathrm{AB}^{\prime \prime}}\right)=0.90,2 J_{\mathrm{AB}^{\prime \prime}} /\left(J_{\mathrm{AB}^{\prime}}+J_{\mathrm{AB}^{\prime \prime}}\right)=1.10$. Since the average $\mathrm{AB}$ superexchange is $J_{\mathrm{AB}}=-4.8 \mathrm{meV}$ in the cubic phase, we arrive at $J_{\mathrm{AB}^{\prime}}=-4.3 \mathrm{meV}$ and $J_{\mathrm{AB}^{\prime \prime}}=-5.3 \mathrm{meV}$.

We calculated the spin wave spectrum from several proposed charge-ordering patterns based on the Cc space group. In the first case, we examined the 11 possible chargeordering patterns that are consistent with Cc symmetry and also satisfy the Anderson condition (of an average of $2.5^{+}$in each tetrahedral cluster of $\mathrm{B}$ sites) ${ }^{34}$ and having $\mathrm{CO}$ wave 


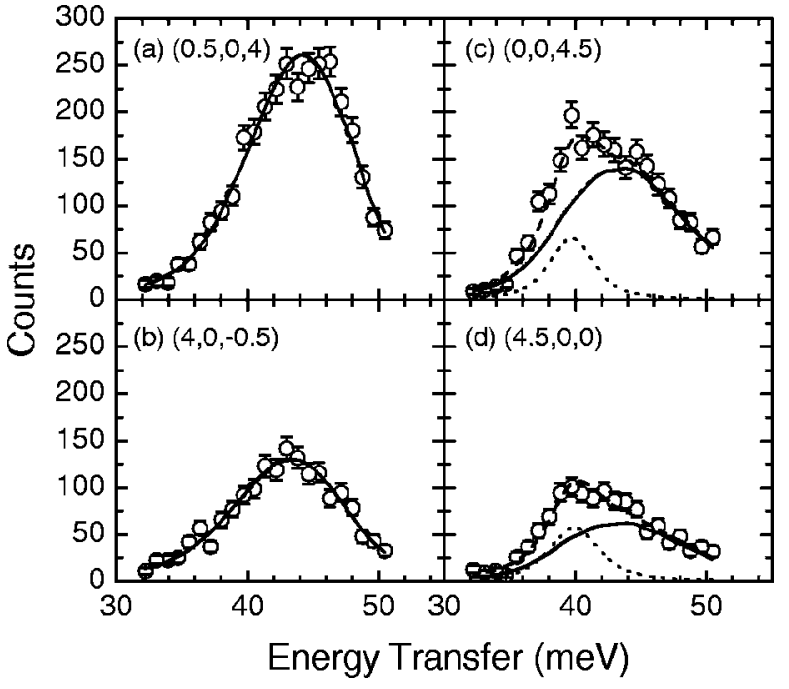

FIG. 16. Transverse [(a) and (b)] and longitudinal [(c) and (d)] constant-Q scans through the acoustic spin wave branch at $\mathbf{q}=(0,0,1 / 2)$ [parallel to magnetic field direction, (b) and (c)] and $(1 / 2,0,0)$ [perpendicular to field, (a) and (b)] above $T_{V}$. Measurements are made in configuration A with a magnetic field of $1 \mathrm{~T}$ applied along the [001] direction. The dashed line is the fit to a spin wave and a phonon excitation using the full triple-axis resolution convolution. The solid line is the spin wave contribution and the dotted line is the phonon contribution to the cross section.

vectors of $(0,0,1 / 2)$. One example of the calculated spin wave dispersion is shown in Fig. 14. There are a large number of spin wave branches (96) arising from the Cc symmetry of the charge ordering pattern. Similar to the atomic distortion model, most of the branches are related to the cubic branches by Brillouin zone folding. None of the charge ordering patterns studied that satisfy the Anderson condition introduce any gap in the acoustic wave.

The second case examined is the pattern obtained from neutron diffraction that has a $\mathrm{CO}$ wavevector of $(0,0,1)$ (called the Wright pattern, after Ref. 5). This charge ordering pattern is also consistent with the Cc space group, but does not satisfy the Anderson condition. The Wright pattern creates the spin wave dispersion shown in Fig. 14(c). The charge ordering pattern in the Wright model does introduce a small gap $(\sim 1 \mathrm{meV})$ in the acoustic mode, suggesting that $\mathrm{CO}$ with $(0,0,1)$ wavevector is necessary to split the acoustic mode. This makes sense, since folding of the Brillouin zone due to (001) type ordering will create a new Brillouin zone boundary at $(0,0,1 / 2)$. However, in our model this splitting is very small compared to the observed splitting of $7 \mathrm{meV}$ and cannot fully explain the gap.

We also examined several Heisenberg models where the $\mathrm{B}-\mathrm{B}$ superexchange was varied according to the different combinations in the charge ordered state; B'-B', B"-B", and B'-B'. Even though the BB superexchange is an order of magnitude smaller than the $\mathrm{AB}$ superexchange, it was anticipated that the spin wave spectrum would be more sensitive to $J_{\mathrm{BB}}$ in the charge-ordered state, since charge ordering occurs on the B sublattice. No such sensitivity was found for the acoustic branch, although models show that the variation of BB superexchange did lift the degeneracy of optical spin

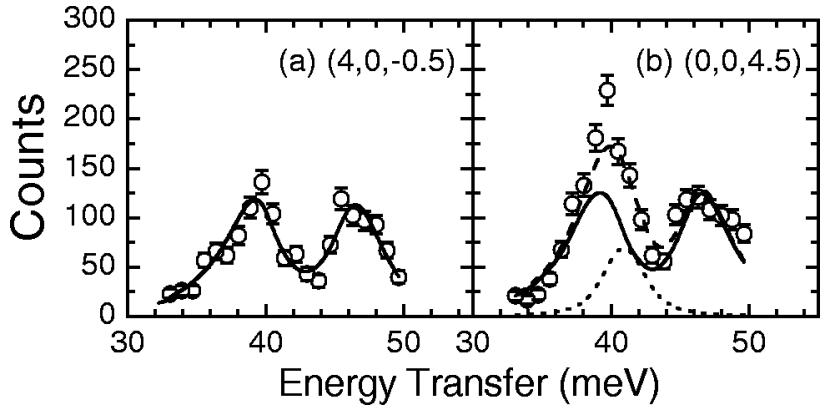

FIG. 17. (a) Transverse and (b) longitudinal scans through the spin wave mode below $T_{V}$ in configuration A. Convolution fits are shown for the spin wave (solid), phonon (dotted) and total intensity (dashed). The lower branch of the spin wave moves down to the energy of the optical phonon below $T_{V}$.

waves propagating on the $\mathrm{B}$ sublattice (in the range of $70-80 \mathrm{meV})$.

\section{Spin-phonon coupling}

We have preliminary evidence that the splitting may be formed from the mixing of the acoustic spin wave with a longitudinal phonon. At high temperatures, we observed a longitudinal optical phonon with energy $\sim 40 \mathrm{meV}$. As shown in Fig. 16, extra intensity appears in the longitudinal scans. This phonon branch can be tracked back to the Brillouin zone center with an energy of $43 \mathrm{meV} .{ }^{39}$ When the phonon branch crosses the spin wave, there is an enhancement of the phonon structure factor, indicating some mixing (not shown). Below $T_{V}$, Fig. 17 shows that the spin wave mode at $(0,0,1 / 2)$ splits, with the lower mode approximately locking in at the energy of the longitudinal optical phonon. We are in the preliminary stages of the study of this effect, and further experimental work to confirm the mixing of these modes is underway.

\section{DISCUSSION}

\section{A. Relation to other materials}

The splitting of the acoustic spin wave branch below $T_{V}$ is a large effect in magnetite. Similar splittings in the acoustic spin waves have been observed in other systems, such as $\mathrm{UO}_{2},{ }^{35} \mathrm{FeF}_{2},{ }^{36} \mathrm{La}_{1-x} \mathrm{Ca}_{x} \mathrm{MnO}_{3},{ }^{37}$ and $\mathrm{YVO}_{3}{ }^{38} \mathrm{In} \mathrm{UO}_{2}$ and $\mathrm{FeF}_{2}$, splittings of the acoustic spin wave branch are of order $1 \mathrm{meV}$. These splittings do not appear in concert with a structural phase transition, but are understood to originate from mixing of an acoustic spin wave with a transverse acoustic phonon. In $\mathrm{La}_{1-x} \mathrm{Ca}_{x} \mathrm{MnO}_{3}$, many splittings of the acoustic spin wave branch are observed that evolve continuously upon cooling. Such splittings have been attributed to a combination of charge ordering and magnon-phonon coupling. In $\mathrm{YVO}_{3}$, a large (5 meV) splitting of the acoustic spin wave branch is observed after a first-order magnetostructural transition. This transition also causes a large decrease in the spin wave bandwidth. It is proposed that these effects on the spin wave spectrum originate from orbital fluctuations/ ordering. The results for $\mathrm{La}_{1-x} \mathrm{Ca}_{x} \mathrm{MnO}_{3}$ and $\mathrm{YVO}_{3}$ are simi- 
lar to the observations discussed here in magnetite. Despite the similarities with magnetite, these other results are discussed in terms of different physical models and the effect of symmetry lowering on the spin waves has not been ruled out.

\section{B. Implications for charge ordering in magnetite}

It is a topical question to ask whether charge ordering even exists in magnetite. ${ }^{7,8}$ From our results, the observation of a gap in the acoustic spin wave at $(0,0,1 / 2)$ can be interpreted as originating from $\mathrm{CO}$ with a wavevector of (001). However, our best attempts to reproduce the size of the splitting from simple arguments concerning the modification of superexchange due to $\mathrm{CO}$ does not predict a large enough gap. Despite our simple estimates (by assuming only $180^{\circ}$ $\mathrm{Fe}-\mathrm{O}-\mathrm{Fe}$ bond angles), we still feel that we are overestimating the superexchange variation. Other than the $(0,0,1 / 2)$ splitting, the majority of other spin wave branches are not very different above and below $T_{V}$ (this is true even of the optical spin wave branches). ${ }^{39}$ The larger superexchange variations required to produce a bigger gap would also strongly influence the rest of the spectrum. This is not observed. Thus, it is unlikely that detailed calculations of the superexchange will produce the right size splitting and not affect other spin wave energies away from $(0,0,1 / 2)$. In other words, the gap appears to be associated with the $(0,0,1 / 2)$ wavevector, thus more likely originating with the coupling to a phonon or charge-density-wave with a specific wavevector. Furthermore, recent experiments have shown that there is probably not full charge disproportionation on the B sublattice, rather the valence probably varies from $2.4^{+}$to $2.6^{+}$ site to site. ${ }^{5,6}$ Thus, the main factor determining the variation of the superxchange due to charge ordering in the ionic model, $\left(1 / S_{i} S_{j}\right)$ in Eq. (27), is suppressed by covalency. This concurs with neutron diffraction data that see only small variations in the magnetic moment sizes in the Verwey phase. ${ }^{5,15,40} \mathrm{We}$ are left to the conclusion that such a large spin wave splitting cannot originate from charge ordering in a purely ionic model. Of course, this does not disprove the existence of charge ordering, but rather implies that the splitting has other origins.

In an itinerant electronic model for magnetite, the Verwey phase can be viewed as the formation of a commensurate charge-density wave (CDW). Neutron diffraction data ${ }^{5}$ and $\mathrm{x}$-ray diffuse scattering data ${ }^{41}$ infer that a CDW with wavevector (001) is present in the Verwey phase. Such a nesting wavevector is predicted from bandstructure calculations. ${ }^{42}$ As our Heisenberg model studies do indicate that (001)-type ordering will cause a splitting at $(0,0,1 / 2)$, the
CDW mechanism cannot be ruled out. The CDW due to nesting instability will cause an itinerant contribution to $J$, peaking near $\mathbf{q}=(0,0,1 / 2)$, and may not affect the rest of the spin wave spectrum to any large degree. More theoretical studies are necessary to determine if the CDW mechanism can explain the results observed here. This being said, it is unlikely that magnetite's magnetic properties should be treated as an itinerant electron system (as opposed to local) since the opening of the electronic gap below $T_{V}$ (Ref. 43) does not profoundly affect the bulk magnetic properties or strongly affect the size of $J_{\mathrm{AB}}$.

\section{Summary}

In summary, we have observed a large gap in the acoustic spin wave branch of magnetite below the Verwey transition temperature. In the monoclinic phase, the spin waves will be affected by charge ordering and small crystalline distortions, because both of these modify the superexchange. By studying Heisenberg models with large unit cells containing modified pairwise superexchange values, we have found that some models do produce small gaps $(\sim 1 \mathrm{meV})$ in the acoustic spin wave at $(0,0,1 / 2)$ [Pmca crystallographic distortions and the (001)-type $\mathrm{CO}$ pattern], but none reproduce the rather large $7 \mathrm{meV}$ gap observed experimentally. It seems necessary that ordering must have a wavevector of (001) in order to split the acoustic mode at $(0,0,1 / 2)$. Better estimates of the magnetic superexchange in the low-temperature charge ordered phase are welcome. However, other than the $(0,0,1 / 2)$ splitting, the majority of other spin wave branches really do not change above and below $T_{V},{ }^{39}$ signifying that the superexchange energy is relatively insensitive to the transition. Thus, it is unlikely that detailed calculations of the superexchange will produce the right size splitting and not affect other spin wave energies away from $(0,0,1 / 2)$. Other origins of the spin wave splitting are possible, such as charge-density wave formation or a large magnetoelastic coupling [i.e. the mixing of a phonon and spin wave near $(0,0,1 / 2)]$.

\section{ACKNOWLEDGMENTS}

The authors would like to thank T. Holden and B. Harmon for useful comments and Zin Tun expert assistance during the Chalk River experiments. Work is supported by the U. S. Department of Energy Office of Science under the following contracts; Ames Laboratory under Contract No. W-7405ENG-82, Oak Ridge National Laboratory, which is managed by UT-Batelle LLC, under Contract No. DE-AC00OR22725 and Brookhaven National Laboratory under Contract DEAC02-98CH10886.

\footnotetext{
*Corresponding author. E-mail: mcqueeney@ameslab.gov

${ }^{1}$ E. J. W. Verwey, Nature (London) 144, 327 (1939).

${ }^{2}$ E. J. W. Verwey, P. W. Haayman, and C. W. Romeijn, J. Chem. Phys. 15, 181 (1947).

${ }^{3}$ M. Iizumi and G. Shirane, Solid State Commun. 17, 433 (1975);

G. Shirane, S. Chikazumi, J. Akimitsu, K. Chiba, M. Matsui, and Y. Fujii, J. Phys. Soc. Jpn. 39, 949 (1975).

${ }^{4}$ J. M. Zuo, J. C. H. Spence, and W. Petuskey, Phys. Rev. B 42, 8451 (1990).

${ }^{5}$ J. P. Wright, J. P. Attfield, and P. G. Radaelli, Phys. Rev. Lett. 87,
} 
266401 (2001); J. P. Wright, J. P. Attfield, and P. G. Radaelli, Phys. Rev. B 66, 214422 (2002).

${ }^{6}$ J. Garcia, G. Subias, M. G. Proietti, H. Renevier, Y. Joly, J. L. Hodeau, J. Blasco, M. C. Sanchez, and J. F. Berar, Phys. Rev. Lett. 85, 578 (2000); J. Garcia, G. Subias, M. G. Proietti, J. Blasco, H. Renevier, J. L. Hodeau, and Y. Joly, Phys. Rev. B 63, 054110 (2001); G. Subías, J. García, J. Blasco, M. Grazia Proietti, H. Renevier, and M. Concepción Sánchez, Phys. Rev. Lett. 93, 156408 (2004).

${ }^{7}$ F. Walz, J. Phys.: Condens. Matter 14, R285 (2002).

${ }^{8}$ J. Garcia and G. Subias, J. Phys.: Condens. Matter 16, R145 (2004).

${ }^{9}$ B. N. Brockhouse, Phys. Rev. 106, 859 (1957); H. Watanabe and B. N. Brockhouse, Phys. Lett. 1, 189 (1962); B. N. Brockhouse and H. Watanabe, in Inelastic Scattering of Neutrons in Solid and Liquids, 1962, edited by E. Eklund (International Atomic Energy Agency, Vienna, Austria, 1963), Vol. II, p. 297.

${ }^{10}$ M. L. Glasser and Frederick J. Milford, Phys. Rev. 130, 1783 (1963). Our definition of the Heisenberg exchange energies $J_{\mathrm{AB}}$ and $J_{\mathrm{BB}}$ is larger than the definition used in this paper by a factor of two.

${ }^{11}$ R. J. McQueeney, M. Yethiraj, W. Montfrooij, J. S. Gardner, P. Metcalf, and J. Honig, J. Appl. Phys. 97, 10A902 (2005).

${ }^{12}$ M. Iizumi, T. F. Koetzle, G. Shirane, S. Chikazumi, M. Matsui, and S. Todo, Acta Crystallogr. 38, 2121 (1982).

${ }^{13}$ H. R. Harrison, R. Aragón, J. E. Keem, and J. M. Honig in Inorganic Synthesis, edited by Smith L. Holt (Wiley, New York, 1982), Vol. 22, pp. 43 ff.

${ }^{14}$ J. P. Shepherd and C. J. Sandberg, Rev. Sci. Instrum. 55, 1696 (1984).

${ }^{15}$ R. Aragon, P. M. Gehring, and S. M. Shapiro, Phys. Rev. Lett. 70, 1635 (1993).

${ }^{16}$ B. H. Torrie, Solid State Commun. 5, 715 (1967).

${ }^{17}$ W. J. L. Buyers, T. M. Holden, E. C. Svensson, R. A. Cowley, and M. T. Hutchings, J. Phys. C 4, 2139 (1971).

${ }^{18}$ M. Eibschutz, U. Ganiel, and S. Shtrikman, Phys. Rev. 151, 245 (1966)

${ }^{19}$ J. B. Goodenough, Phys. Rev. 171, 466 (1968).

${ }^{20}$ J. W. Lynn, H. A. Mook, and W. J. L. Buyers, Phys. Rev. B 12, 238 (1975)
${ }^{21}$ K. P. Belov, A. N. Goryaga, and Kh. Sh. Borlakov, Sov. Phys. Solid State 29, 2106 (1986).

${ }^{22}$ Z. Zhang and S. Satpathy, Phys. Rev. B 44, 13319 (1991).

${ }^{23}$ V. N. Antonov, B. N. Harmon, V. P. Antropov, A. Ya. Perlov, and A. N. Yaresko, Phys. Rev. B 64, 134410 (2001).

${ }^{24}$ C. M. Srivastava and R. Aiyar, J. Phys. C 20, 1119 (1987).

${ }^{25}$ R. Aragon, Phys. Rev. B 46, 5334 (1992), and references therein.

${ }^{26}$ A. W. Saenz, Phys. Rev. 125, 1940 (1962).

${ }^{27}$ M. J. Cooper and R. Nathans, Acta Crystallogr. 23, 357 (1967).

${ }^{28} \mathrm{~A}$. Zheludev, RESLIB program, 〈http://neutron.ornl.gov/ zhelud/reslib/index.html〉.

${ }^{29}$ S. K. Mishra and S. Satpathy, Phys. Rev. B 58, 7585 (1998); H. Meskine, H. Konig, and S. Satpathy, ibid. 64, 094433 (2001).

${ }^{30} \mathrm{~W}$. A. Harrison, Electronic Structure and the Properties of Solids (Dover, New York, 1989).

${ }^{31}$ P. W. Anderson, Phys. Rev. 115, 2 (1959).

${ }^{32}$ C. M. Srivastava, G. Srinivasan, and N. G. Nanadikar, Phys. Rev. B 19, 499 (1979).

${ }^{33}$ P. G. Bercoff and H. R. Bertorello, J. Magn. Magn. Mater. 169, 314 (1997)

${ }^{34}$ P. W. Anderson, Phys. Rev. 102, 1008 (1956).

${ }^{35}$ R. A. Cowley and G. Dolling, Phys. Rev. 167, 464 (1968); R. Caciuffo, G. Amoretti, P. Santini, G. H. Lander, J. Kulda, and P. deV. DuPlessis, Phys. Rev. B 59, 13892 (1999).

${ }^{36}$ B. D. Rainford, J. G. Houmann, and H. J. Guggenheim, in Neutron Inelastic Scattering 1972 (International Atomic Energy Agency, Vienna, Austria, 1972), p. 655.

${ }^{37}$ M. Hennion and F. Moussa, New J. Phys. 7, 84 (2005).

${ }^{38}$ C. Ulrich, G. Khaliullin, J. Sirker, M. Reehuis, M. Ohl, S. Miyasaka, Y. Tokura, and B. Keimer, Phys. Rev. Lett. 91, 257202 (2003).

${ }^{39}$ R. J. McQueeney (unpublished).

${ }^{40}$ C. G. Shull, E. O. Wollan, and W. C. Koehler, Phys. Rev. 84, 912 (1951).

${ }^{41}$ K. Siratori, Y. Ishii, Y. Morii, S. Funahashi, S. Todo, and A. Yanase, J. Phys. Soc. Jpn. 67, 2818 (1998).

${ }^{42}$ A. Yanase and K. Siratori, J. Phys. Soc. Jpn. 68, 1607 (1999).

${ }^{43}$ S. K. Park, T. Ishikawa, and Y. Tokura, Phys. Rev. B 58, 3717 (1998). 